\title{
LA UNIÓN DE LA ENERGÍA Y LA GOBERNANZA DEL SISTEMA TIERRA EN EL ANTROPOCENO: UNA CUESTIÓN CONSTITUCIONAL.
}

\author{
PROF. DR. ENDRIUS COCCIOLO \\ Centre d'Estudis de Dret Ambiental de Tarragona (CEDAT) \\ Universitat Rovira i Virgili \\ endrius.cocciolo@urv.cat
}

"El sector de la energía ha quedado demasiado tiempo al margen de las libertades fundamentales de nuestra Unión".

J.C. JUNCKER, presidente de la Comisión Europea, 25 de febrero de 2015

"Es preciso estudiar el poder fuera del modelo del Leviatán, fuera del campo delimitado por la soberanía jurídica y la institución estatal".

FOUCAULT, M., Genealogía del racismo, La Piqueta, Madrid, 1992, p. 43

"[...] necesitamos cambiar el modelo de desarrollo global, lo cual implica reflexionar responsablemente sobre el sentido de la economía y su finalidad, para corregir sus disfunciones y distorsiones. No basta conciliar, en un término medio, el cuidado de la naturaleza con la renta financiera, o la preservación del ambiente con el progreso. En este tema los términos medios son sólo una pequeña demora en el derrumbe"

SANTO PADRE FRANCISCO, Laudato si', carta encíclica sobre el cuidado de la casa común, 2015, p. 148

"In the Anthropocene, the metrics of economic growth or 'sustainable development' are unsustainable".

ROBINSON, N.A., "Fundamental Principles of Law for the Anthropocene?", Environmental Policy and Law, 44/1-2, 2014, p. 17

\footnotetext{
* El presente artículo ha sido realizado en el marco del proyecto de investigación "Del desarrollo sostenible a la justicia ambiental: hacia una matriz conceptual para la gobernanza global", Ministerio de Economía y Competitividad, ref. DER2013-44009-P, 2014-2016, IP: Prof. Dr. Antoni Pigrau.
} 
Recibido: 21 de junio de 2015 / Aceptado: 29 de junio de 2015

RESUMEN: La transformación de las estructuras políticas y jurídicas que plantea la nueva estrategia europea para la Unión de la Energía está relacionada con las dinámicas de constitucionalización que emergen en el fragmentado panorama de los regímenes de gobernanza del capitalismo global. El estudio analiza si la estrategia desarrolla un nuevo paradigma constitucional o si persiste en un modelo de constitución económica material cuyo objetivo esencial es la construcción de un espacio homogéneo para asegurar el libre flujo energético. Sin embargo, las cuestiones constitucionales de la energía, del medio ambiente o de la economía, vistas a través de las lentes del antropoceno, brindan una nueva comprensión de las relaciones entre naturaleza y sociedad, y, por ende, un cambio hacia una nueva gubernamentalidad y la reconsideración de la visión del espacio sociopolítico y jurídico de la modernidad. El nuevo constitucionalismo ecosistémico establece límites a los poderes privados del capitalismo avanzado y articula las garantías institucionales contra las amenazas de las matrices anónimas a los sistemas planetarios.

RESUM: La transformació de les estructures polítiques i jurídiques que planteja la nova estratègia europea per a la Unió de l'Energia està relacionada amb les dinàmiques de constitucionalització que emergeixen en el fragmentat panorama dels règims de governança del capitalisme global. L'estudi analitza si l'estratègia desenvolupa un nou paradigma constitucional o si persisteix en un model de constitució econòmica material l'objectiu essencial del qual és la construcció d'un espai homogeni per assegurar el lliure flux energètic. No obstant això, les qüestions constitucionals de l'energia, del medi ambient o de l'econòmica, vistes a través de les lents del Antropocè, brinden una nova comprensió de les relacions entra naturalesa i societat, per tant un canvi cap a una nova governamentalitat i la reconsideració de la visió de l'espai socio-polític i jurídic de la modernitat. El nou constitucionalisme ecosistèmic estableix límits als poders privats del capitalisme avançat $\mathrm{i}$ articula les garanties institucionals contra les amenaces de les matrius anònimes als sistemes planetaris. 
ABSTRACT: The transformation of the political and legal structures posed by the new European strategy for an Energy Union is related to the dynamics of constitutionalisation, raised from the fragmented landscape of governance regimes of global capitalism. The article analyzes if the strategy develops a new constitutional paradigm or if it continues on the path of a material economic constitution whose main objective is the construction of a homogeneous space to ensure the free flow of energy. However, the constitutional issues of energy, environment or economy, seen through the lens of the Anthropocene, provide a new understanding of the relationship between nature and society, and therefore a shift to a new governmentality and the reconsideration of the vision of socio-political and legal space of modernity. The new ecosystemic constitutionalism sets limits to the private power of advanced capitalism and articulates the institutional guarantees against threats from anonymous matrices to the planetary systems.

PALABRAS CLAVE: Unión de la Energía; política energética europea; gobernanza global; gobernanza del sistema Tierra; derecho de la energía; justicia energética; constitucionalismo global; antropoceno; sostenibilidad; teoría de sistemas.

PARAULES CLAU: Unió de l'Energia; Política energètica europea; Governança global; Governança del Sistema Terra; Dret de l'Energia; Justícia energètica; Constitucionalisme global; Antropocè; sostenibilitat; Teoria de sistemes.

KEYWORDS: Energy Union; European Energy Policy; Global Governance; EarthSystem Governance; Energy Law; Energy Justice; Global Constitutionalism; Anthropocene; Sustainability; Systems Theory. 
SUMARIO: I. Introducción. La centralidad de la cuestión energética. II. La energía como problema de justicia en el sistema del capitalismo global y su necesaria constitucionalización. III. El trilema de la energía en Europa. 1. Las dimensiones de la cuestión energética. 2. Los fundamentos teóricos de la política energética europea. 3. La integración energética europea entre mercado y desarrollo sostenible. 4. La europeización de la seguridad y la Unión de la Energía como dispositivo. IV. Consideraciones finales sobre antropoceno, gobernanza, energía y constitucionalismo. V. Bibliografía.

\section{INTRODUCCIÓN. LA CENTRALIDAD DE LA CUESTIÓN ENERGÉTICA}

El 25 de febrero de 2015 la Comisión Europea, mediante comunicación, lanzó la "Estrategia Marco para una Unión de la Energía resiliente con una política climática prospectiva" ; este documento, junto con otras dos comunicaciones en materia de cambio climático $^{2}$ e interconexiones eléctricas ${ }^{3}$, integra el nuevo paquete sobre la Unión de la Energía, cuyo objetivo es provocar "una transformación profunda del sistema energético europeo", especialmente urgente a causa de la vulnerabilidad energética derivada de los eventos geopolíticos a los que está expuesta la $\mathrm{UE}^{4}$. El 18 de mayo de 2015 un estudio publicado por el FMI reveló que las compañías energéticas que explotan recursos fósiles reciben subsidios globales equivalentes a 5,3 billones de dólares anuales, una cantidad superior al total del gasto sanitario de todos los gobiernos mundiales a la que se llega sumando los costos y las externalidades negativas que soportan los Estados por el uso de combustibles fósiles ${ }^{5}$. El 18 de junio de 2015 se publicó la encíclica Laudato si', sobre el cuidado de la casa común, del papa Francisco ${ }^{6}$, en la que se apunta a una conversión ecológica y una transformación cultural para salvar el planeta y la sociedad del uso irresponsable de sus recursos y de los excesos del sistema económico. Resulta evidente que la cuestión energética está en el centro de las agendas políticas globales por sus implicaciones políticas, económicas, ambientales, sociales, tecnológicas y éticas. El presente trabajo analiza los procesos de constitucionalización derivados de las dinámicas de los sistemas supranacionales de

\footnotetext{
${ }^{1} \mathrm{COM}(2015) 80$ final.

${ }^{2}$ COM(2015) 81 final, "El Protocolo de París, un plan rector para combatir el cambio climático más allá de 2020 ".

${ }^{3} \operatorname{COM}(2015) 82$ final, "Alcanzar el objetivo de interconexión de electricidad del 10 \%. Preparación de la red eléctrica europea de 2020".

${ }^{4} \operatorname{COM}(2015) 80$ final.

${ }^{5}$ Vid. COADY, D., PARRY, I., SEARS, L. y SHANG, B., “How Large Are Global Energy Subsidies?”, IMF Working Paper No. 15/105, $2015 . \quad$ Disponible http://www.imf.org/external/pubs/ft/wp/2015/wp15105.pdf

${ }^{6}$ Carta encíclica Laudato si' del Santo Padre Francisco sobre el cuidado de la casa común. Disponible en: http://ep00.epimg.net/descargables/2015/06/18/a039ba1c2b0e3a9d3d24380c8e762116.pdf
} 
gobernanza de la energía en el marco del antropoceno, la nueva época planetaria que exige la reconsideración de la visión de los espacios sociopolíticos y jurídicos de la modernidad. El trabajo se estructura en tres partes: en la primera se establecen las relaciones entre cuestión energética como problema de justicia, constitucionalismo y crisis globales en el marco político del antropoceno, siguiendo la teoría crítica postsistémica de Teubner; en la segunda parte se analiza el "trilema" de la energía en Europa —economía, sostenibilidad, seguridad - desde los tratados fundacionales hasta la reciente estrategia de la Unión de la Energía, poniendo de manifiesto la emersión de una constitución económica material de la energía vinculada a la gubernamentalidad neoliberal; y la tercera parte contiene reflexiones finales al hilo del nuevo marco epistémico que suponen el antropoceno y los retos para el constitucionalismo global.

\section{LA ENERGÍA COMO PROBLEMA DE JUSTICIA EN EL SISTEMA DEL CAPITALISMO GLOBAL Y SU NECESARIA CONSTITUCIONALIZACIÓN}

Con la estrategia para la Unión de la Energía la cuestión energética vuelve a estar en el centro del proceso de la integración europea seis décadas después de la firma de los tratados de París (CECA, 1951) y Roma (CEE y EURATOM, 1957). Se trata de un reajuste urgente y al mismo tiempo problemático que plantea una cuestión compleja: si la estrategia de europeización de la energía responde a la necesidad de desarrollar un nuevo paradigma ${ }^{7}$ para la gobernanza ultraestatal e intergeneracional de un sector clave para la continuidad social, asegurando un "acoplamiento constitucional" entre sistemas sociales y sistema ecológico, o si, en cambio, tiene como objetivo la construcción de un espacio homogéneo para propiciar la expansión de los sistemas energético y económico. Se trata de una cuestión fundamental para cualquier intento sólido de hacer frente a las crisis energéticas y ecológicas en curso y para superar las brechas jurídicas existentes. Esta aproximación implica ir más allá de las preguntas convencionales en materia de clima y energía. En este sentido, Sovacool afirma:

\footnotetext{
${ }^{7}$ Sobre la importancia del cambio de paradigma, especialmente en el ámbito de la energía, vid. GOlThaU, A. y SITTER, N., The Power of Paradims: the EU and the Global Energy Policy, 2012. Disponible en: http://ssrn.com/abstract=1986832

${ }^{8}$ Sobre el concepto de "acoplamiento constitucional" o constitutional bonding, sobre el que volveremos más adelante, vid. KJAER, P.F., "Law nad Order whitinh and beyond National Configurations", Normative Orders Working Papers, 2/2010, pp. 1-48. Disponible en http://ssrn.com/abstract=1687013
} 
Influenced by an obsession with economics and technology, most energy and climate analysts frequently ask the wrong questions. They will ponder how large proven reserves of oil and gas are being extracted rather than challenging the need to utilize oil and gas in the first place, or asking whether oil and gas infrastructures are fair to their workers or the communities that live near them. They will assess and model energy prices and technological learning curves, rather than ask how existing energy infrastructures benefit some people to the exclusion of others. They will map energy and climate scenarios, track atmospheric concentrations of carbon dioxide, and discuss the pros or pitfalls of the UNFCCC, but refrain from interrogating the underlying logic behind an international economic system that continues to emit dangerous amounts of greenhouse gases ${ }^{9}$.

Los problemas de seguridad energética y acceso a la energía no solo se sitúan en la encrucijada de los interrogantes éticos difíciles de resolver, sino que la cuestión energética determina uno de los temas fundamentales para la justicia de nuestros tiempos. De acuerdo con Sovacool y Dworkin:

Clearly, we need new ways of thinking about, and approaching, the world's energy problems and the issues at hand make global energy security and access among the central justice issues of our time, with profound implications for happiness, welfare, freedom, equity, and due process ${ }^{10}$.

Las decisiones de política energética no pueden, por lo tanto, limitarse solo a asegurar la correspondencia entre suministro y demanda, sino que deben tener en cuenta una compleja combinación de elementos económicos, sociales, políticos, ecológicos, tecnológicos, etc. Además, la dificultad de la cuestión energética resulta, pues, de la imbricación de cuatro factores: la afectación interjurisdiccional, la dimensión intergeneracional, la escasez de los recursos comunes y la policontextualidad social. En efecto, la generación y el uso de la energía se enfrentan a retos específicos propios de cada uno de los subsistemas sociales de una sociedad caracterizada por un elevado grado de complejidad funcional. En el sistema económico el desafío es el crecimiento; en el sistema político, la independencia energética; para el propio sistema energético, el reto es la continuidad del suministro; mientras que, para el sistema ecológico, el problema es la sostenibilidad. Desde la década de 1980 se han llevado a cabo muchos

\footnotetext{
${ }^{9}$ SOVACOOL, B., "Exposing the Paradoxes of Climate Change and Energy Governance", International Studies Review, 16, 2014, p. 294, (el énfasis es nuestro).

${ }^{10}$ SOVACOOL, B. y DWORKIN, M., "Energy Justice: Conceptual Insights and practical applications", Applied Energy, vol. 142, 2015, p. 436. Para un desarrollo más amplio del tema, vid., de los mismos autores, Global Energy Justice: Problems, Principles, and Practice, Cambridge University Press, 2014.
} 
esfuerzos para coordinar los sistemas sociales bajo el discurso del desarrollo sostenible. Como veremos más adelante, se trata, sin embargo, de esfuerzos aparentemente neutrales que encubren una expansión de la racionalidad propia de los sistemas económicos y energéticos que, con el soporte del derecho y de la política y sin la imposición de los límites necesarios, conduce a resultados dañinos para la sociedad y el planeta en su conjunto ${ }^{11}$.

Para el derecho surge entonces el problema de generar contrainstituciones para proteger la reproducción social. Esta demanda normativa no parece nueva, en efectos, la función del sistema jurídico en efectos es la de operar como "sistema inmunitario de la sociedad"12 y la respuesta, en términos de herramientas jurídicas para la estabilización social, en su nivel más alto, ha asumido tradicionalmente la forma de las constituciones. Sin embargo, el constitucionalismo que se está desarrollando en la época actual es un constitucionalismo pluralista, más allá de los Estados, conectado con los sistemas sociales del capitalismo globalizado, que a su vez está "integrado" en el sistema tierra, condicionando los ecosistemas de tal manera que el planeta habría entrado en una nueva era geológica denominada antropoceno ${ }^{13}$ :

\footnotetext{
${ }^{11}$ Parenteau nos recuerda la dramática evidencia de los datos: "By almost any measure, we are exceeding the earth's carrying capacity. Human population, currently numbering seven billion and projected to hit nine billion by mid-century, coupled with a rapidly rising per capita consumption rate underlie all of the other present drivers of global change. Though humans make up less than one-half of one percent of the global biomass, we use up $25-32 \%$ of the earth's net primary productivity. Humans have converted $43 \%$ of land to agricultural or urban landscapes, with much of the remaining natural landscape fragmented by roads and utilities. This exceeds the physical transformation that occurred at the last global-scale critical transition when $30 \%$ of the earth's surface went from being covered by glacial ice to being ice-free. With extinction rates already 100 to 1,000 times background rates, and projected to increase dramatically in response to anthropogenic global warming, humans are literally altering the course of evolution. Speaking of climate change, atmospheric concentrations of $\mathrm{CO} 2$ have increased by $39 \%$ since the Industrial Revolution and, at approximately $400 \mathrm{ppm}$, are now the highest in 15 million years. We are adding 2.2 ppm per year. At this rate, worldwide CO2 levels will substantially exceed 1,000 ppm by the end of this century. The level of heating that would result from this degree of concentration would be beyond anything seen during any period in which earth supported complex life", vid. PARENTEAU, P., "It's the Biosphere, Stupid", Burger, M. et ál., Rethinking Sustainability to Meet the Climate Change Challenge, en Environmental Law Reporter, vol. 43, 2012, p. 10347, disponible en http://ssrn.com/abstract=2189530.

${ }^{12}$ PRANDINI, R., "La costituzione del diritto nell'epoca della globalizzazione. Struttura del societàmondo e cultura del diritto nell'opera di Gunther Teubner", Teubner, G., La cultura del diritto nell'epoca della globalizzazione. L'emergere delle costituzioni civili, Armando Editore, p. 198.

${ }^{13}$ El término antropoceno fue acuñado por el premio Nobel Paul Crutzen e identifica una nueva época en la historia planetaria caracterizada por la alteración de los ecosistemas terrestres a causa de las actividades humanas. La Comisión Internacional de Estratigrafía decidirá en 2016 si el antropoceno es formalmente reconocido como nueva época geológica después del holoceno. Vid. CRUTZEN, P. y STOERMER, E. F., The Anthropocene. IGBP Newsletter, 41, 2000, pp. 17-18. Disponible en: http://www.igbp.net/download/18.316f18321323470177580001401/NL41.pdf
} 
In the Anthropocene, ecosystems are not just external constraints on human activity. We are not just in the system; we also help drive its parameters. So what makes the Anthropocene different is the lack of fixed reference points for collective action given by the desirable state of key systems, and that includes planetary boundaries ${ }^{14}$.

En definitiva, es una nueva demanda de normatividad la que procede de la sociedad del antropoceno y, por lo tanto, no se puede ofrecer una respuesta dentro de los paradigmas jurídicos conocidos. Desde esta perspectiva, abordar los problemas energéticos en términos constitucionales para establecer objetivos y límites requiere entablar una discusión sobre normas y valores para determinar qué fines son aceptables, responsables y equitativos. Al contrario, una aproximación más limitada, centrada, por ejemplo, solo en las alternativas del mix energético, como avisa Becker, podría alejar la identificación de soluciones para lograr un sistema más sostenible a largo plazo ${ }^{15}$.

Ahora bien, los problemas resultantes del uso de la energía, como los conflictos por los recursos, el calentamiento global, la alteración química de los océanos, la pobreza energética, las amenazas a la salud, etc., no pueden tratarse solo desde la dimensión del individuo. En primer lugar, porque los sistemas morales humanos no están bien equipados para hacer frente a semejante clase de problemas. De ahí la baja resistencia ante los discursos hegemónicos y la modesta realización de cambios sustantivos. Dicha inadecuación depende de la dificultad de captar la dimensión ética que suponen el uso de las energías y los problemas climáticos: los resultados de estudios realizados en neurociencias y ciencias del comportamiento coinciden en demostrar que el sistema humano de juicio moral no está bien dotado para identificar el cambio climático —un fenómeno complejo, a gran escala y no intencional - como un importante imperativo moral $^{16}$. En segundo lugar (y aún más importante), porque los problemas enunciados están ligados a procesos sociales. En concreto, se trata de la "compulsión hacia el

\footnotetext{
${ }^{14}$ DRYZEK, J.S., "Institutions for the Anthropocene: Governance in a Changing Earth System", British Journal of Political Science, abril de 2015, p. 4.

${ }^{15}$ Según Becker, "a narrow focus on finding substitutes for current energy sources or efficient technologies within the existing systems and societal frameworks could prevent us from finding more feasible and substantial solutions for a new future energy system", vid. BECKER, C., Ethical Aspects of the Energy Issue: Toward a Sustainable Energy Future, 2011, p. 3. Disponible en: http://ssrn.com/abstract=1768194

${ }^{16}$ MARKOWITZ, E. M. y SHARIFF, A.F., "Climate change and moral judgement", Nature Climate Change, vol. 2, abril de 2012, pp. 243-247.
} 
crecimiento" 17 tanto del sistema económico como del sistema energético que está en la base de las crisis globales. De hecho, existe una vinculación entre globalización, economía, energía, ecología y crisis. La globalización puede entenderse como una "fase de transición" ${ }^{\text {"18 }}$ del capitalismo industrial fordista al capitalismo financiero globalizado en el que se da la fragmentación de los sistemas sociales (policontextualidad) y del derecho. La financiarización de la economía supone pasar de un sistema de producción, banca y comercio que dio forma a la sociedad moderna a la instauración de un sistema en el que las finanzas se han posicionado como motor y guía del capitalismo ${ }^{19}$. En este segundo modelo, el proceso de acumulación tiene lugar a través de canales financieros antes que por las vías tradicionales de producción y comercio ${ }^{20}$. Este orden económico empezó su desarrollo en la década de 1970 y fue catalizado por la desregulación de las décadas de 1980 y 1990, hasta llegar a su crisis más radical en 2008. El capitalismo financiero tiene al menos dos características esenciales $\left.{ }^{21}: 1\right)$ un enorme incremento en el valor total de los activos financieros que circulan a nivel global como consecuencia de la multiplicación de los instrumentos financieros facilitados por la titularización y los derivados; y 2) el desacoplamiento entre la economía real y la economía financiera, con una desmedida creación de riqueza financiera ficticia. Esta fase ha dado lugar a un crecimiento patológico basado en la deuda, de manera que los eventos de 2008 deben considerarse como el desenlace inevitable de lo que podría denominarse "the great credit super-cycle" ${ }^{22}$. La globalización de los flujos de capitales (en gran medida moneyless gracias a las tecnologías de la comunicación) ha arrebatado de las manos de los bancos centrales nacionales la creación del dinero. Esta función viene ejercida de facto por la banca comercial mediante la provisión de garantías de crédito. Los bancos

\footnotetext{
${ }^{17}$ Para usar la acertada expresión de Teubner, vid. TEUBNER, G., "A Constitutional Moment? The Logic of Hit the Bottom", Kjaer, P., Teubner, G. y Febbrajo, A. (eds.), The Financial Crisis in Constitutional Perspective. The Dark Side of Functional Differentiation, Hart Publishing, 2011, p. 3 y ss.

${ }^{18}$ La idea de la globalización como fase de transición se debe a KJAER, P., "Law and Order within and Beyond National Configurations", Normative Orders Working Paper, núm. 02/2010, p. 30. Disponible en: http://ssrn.com/abstract=1687013

${ }^{19}$ En estos términos se expresa el premio Nobel 2013 Robert Shiller; vid. SHILLER, R., Las finanzas en una sociedad justa, Deusto, Barcelona 2012, p. 20.

${ }^{20}$ Vid. EPSTEIN, G.A., Financialization and The World Economy, Edward Elgar Publishing, 2006.

${ }^{21}$ Vid. BRESSER-PEREIRA, L.C., “The Global Financial Crisis and a New Capitalism?", Levy Economics Institute Working Paper, núm. 592, mayo 12, 2010, p. 8 y ss. Disponible en: $\mathrm{http}: / /$ ssrn.com/abstract $=1605180$

${ }^{22}$ Vid. MORGAN, T., "Perfect Storm. Energy, Finance and the End of Growth", Strategy Insights, issue nine, Tullett Prebon, 2013, p. $5 . \quad$ Disponible en: https://www.tullettprebon.com/Documents/strategyinsights/TPSI_009_Perfect_Storm_009.pdf
} 
centrales influyen solo de forma indirecta en esta función de creación privada de dinero. Teubner afirma:

It is this massive creation of money by private banks that is responsible for the current excesses of the compulsion to growth in the global financial sector. It serves, through advance financing, to compel the real economy to grow to an extent that is socially harmful. At the same time, this private money creation is exploited for an unforeseen increase in self-referential financial speculation ${ }^{23}$.

En este punto es importante establecer el vínculo con la energía. Morgan observa que el dinero es el lenguaje y no la sustancia de la economía ya que, en definitiva, la economía es una ecuación de la energía excedente (“a surplus energy equation”) que depende de las leyes de la termodinámica y no de las leyes del mercado ${ }^{24}$. En este orden de ideas, continúa Morgan del siguiente modo:

The terms "labour" and "energy" are coterminous through the commonality of energy, so anything which could be purchased with money was the product of energy, past, present or future. With the broader term "energy" replacing "labour", exactly the same relationship prevails in the industrial societies of today, except that exogenous energy inputs (overwhelmingly dominated by fossil fuels) now provide the vast majority of the energy used in the economy.

La clave de este discurso, según Morgan, es que, una vez entendida la verdadera función del dinero como sustitución ("tokenisation") de la energía, entonces se puede resituar el concepto de "deuda" en el contexto de la energía. En efecto, dicho de una manera muy básica, una deuda puede ser considerada como una reclamación de dinero en diferido; sin embargo, dado que el dinero es un valor asociado a la energía, de ello se deriva que la deuda es en realidad una reclamación de energía en diferido. Por ello, la viabilidad de la sociedad del capitalismo avanzado, cuyo funcionamiento económico está asentado en el exceso de crédito, depende en definitiva de la disponibilidad de energía en el futuro y, por ende, precisa un equivalente suministro expansivo de energía para satisfacer la compulsión al crecimiento ${ }^{25}$. En palabras de Morgan:

\footnotetext{
${ }^{23}$ TEUBNER, “A Constitutional Moment?...”, cit., p. 4.

${ }^{24}$ Afirma MORGAN: "Society and the economy began when agriculture created an energy surplus which, though tiny by later standards, liberated part of the population to engage in non-subsistence activities", MORGAN, "Perfect Storm...", cit., p. 11.

${ }^{25}$ Ibíd., p. 65.
} 
$[\mathrm{T}]$ he economy is, in reality, an energy dynamic onto which has been grafted not just a system of monetary tokenisation but, much more seriously, a system of anticipatory finance which is viable if (but only if) it can be assumed that there will be no significant check to the process of exponential economic growth. Of course, the most obvious threat to this anticipatory economic system would arise if the availability of energy were to diminish (or even simply cease to increase in the way that anticipatory finance necessarily assumes). Since the 1950s, this threat has acquired a name — "peak oil".

Ahora bien, muchos podrán objetar que la teoría del peak oil de Hubbert es una amenaza fantasma, desmentida por la revolución tecnológica de las energías fósiles convencionales y no convencionales - por ejemplo, la estadounidense- que ponen de manifiesto la amplia disponibilidad de recursos bajo la superficie terrestre, así como por la significativa reducción de los precios del crudo. Este nuevo escenario parece poner en tela de juicio buena parte de los fundamentos de la política energética europea y de la icónica Energiewende alemana ${ }^{26}$. Sin embargo, una vez más, Morgan alerta de que el verdadero problema de la teoría del pico del petróleo no concierne a las reservas disponibles, sino a la cuestión más crítica de la tasa de retorno energético (EROEI, energy returned on energy invested), que muestra un progresivo y acelerado deterioro. Hemos llegado a un punto esencial de nuestro discurso. Al hallazgo de reservas de petróleo de 1930 se asociaba un EROEI de más de 100:1, es decir, un coste de la energía del 0,99\% (1/101). El promedio general de EROEI habría bajado del 40:1 del 1990 al 20:1 de 2010; esto implica que el coste teórico de la energía ha sido del 4,76\% del PIB. Ulteriores deterioros de la ecuación llevarían a una caída radical de los beneficios y a un aumento exponencial de los $\operatorname{costes}{ }^{27}$. De mantenerse esta tendencia, la caída del retorno energético obligaría a que una parte cada vez mayor de energía generada tendrá que ser empleada para explotar los recursos energéticos, reduciendo el valor destinado a otros usos; a la vez, esta dinámica conlleva efectos perniciosos para la sociedad más allá del aumento de los costes económicos, por ejemplo en términos de degradación ambiental.

En resumen, en la sociedad del capitalismo avanzado, los procesos de globalización producen la emancipación de los sistemas sociales (economía, política, derecho,

\footnotetext{
${ }^{26}$ Vid. HELM, D., "The price of oil", Energy Future Network, Paper no. 6, 3 de diciembre de 2014. Disponible en: http://www.dieterhelm.co.uk/sites/default/files/The\%20price\%20of\%20oil.pdf

${ }^{27}$ Para conocer con más detalle los datos y argumentos expuestos, vid. MORGAN, "Perfect Storm...", cit., p. 65 y ss.
} 
energía, etc.) respecto de las bases territoriales de los Estados y de su soberanía. Los sistemas sociales se caracterizan por racionalidades específicas y dinámicas de crecimiento. En especial, la financiarización del sistema económico capitalista ha provocado una compulsión al crecimiento cuyos efectos nocivos, aunque evidentes en repetidas crisis cíclicas $^{28}$, se han manifestado con máxima virulencia en la crisis financiera de 2008 y en su posterior transformación en la crisis de la deuda soberana que ha afectado a Europa especialmente a partir del 2010. El acoplamiento del sistema económico con el sistema energético ha impulsado en este segundo una mayor oferta de energía (cuyo exceso ha dado lugar a una abrupta caída del precio del petróleo). Esta mayor generación de energía daba valor a una mayor creación de dinero a través del crédito $^{29}$. Sin embargo, la explotación de recursos energéticos se caracteriza por un decrecimiento de la tasa de retorno energético respecto de la energía invertida para su extracción. Los análisis desarrollados desde el ámbito de la economía ecológica terminan de explicar cómo los factores ecológicos están relacionados con la crisis económica:

Debts increase exponentially. They can be paid by economic growth, by inflation or by squeezing the debtors for a while. But economic growth of the productive economy depends on energy and materials, and on the availability of sinks for waste such as carbon dioxide. We see the building industry or car manufacturing as part of the "productive" economy but they depend on exhaustible resources. "The entropy law and the economic process" by Georgescu-Roegen (1971) insisted in the fact that energy cannot be recycled, and that materials are recycled only to some extent. Fresh supplies are needed, and this is problematic when we rely on exhaustible fossil fuels and on materials which are ever more difficult to obtain at the commodity frontiers. The fictitious economy of finance issues money and debt to keep up the required nominal growth rates that cannot be sustained by the ecological economy. As debt accumulates, a crisis ensues, manifested in

\footnotetext{
${ }^{28}$ Los economistas han puesto de manifiesto que las crisis económicas y financieras están lejos de ser un fenómeno raro; en este sentido, téngase en consideración el cómputo de las crisis ocurridas entre el 1622 y la crisis de las subprime: nueve hasta el 1700, 22 en el siglo XIX hasta la época del "patrón oro", siete entre el 1880 y el 1913, ocho en la etapa entre las dos guerras mundiales (1919-1939), seis durante la época del Bretton Woods y, finalmente, dieciséis entre el 1974 y el 2008. Las evidencias sugieren que las crisis ocurren con la suficiente frecuencia como para considerarlas como algo consustancial al capitalismo. Vid. FRATIANNI, M., Financial Crisis, Safety Net and Regulation, Invited lecture at the Annual meeting of the Società Italiana degli Economisti (Perugia, 24 de octubre de 2008), p. 3. Disponible en: http://papers.ssrn.com/sol3/papers.cfm?abstract_id=1286903

${ }^{29}$ DOUTHWAITE, R., "Degrowth and the supply of money in an energy-scarce world", Ecological Economics, vol. 84, 2012, pp. 187-193.
} 
demands to pay debts through inflation (expansion) or by squeezing the debtors (austerity) $)^{30}$.

Se ha puesto también de manifiesto que existe un riesgo financiero global relacionado con la burbuja del carbono en los mercados de capitales. Según Legget, cuando los analistas convierten las reservas de combustibles fósiles en un equivalente de emisiones de dióxido de carbono y comparan esta medida total con el budget global de carbono disponible para mantener el calentamiento global por debajo del umbral de los dos grados de media, se llega a una conclusión perturbadora: hay un abrumador exceso de carbono que no puede ser quemado si no se quiere rebasar los dos grados. Por lo tanto, se manifiesta una disyuntiva dramática: o se va a incumplir estrepitosamente el objetivo de mantener el calentamiento global por debajo de los dos grados de media y, por lo tanto, la lucha contra el cambio climático está perdida, o las 100 principales empresas energéticas mundiales, cuyo valor alcanza casi los 7,5 billones de dólares, están asentadas en una burbuja cuya explosión revertería en otra crisis financiera global ${ }^{31}$.

En pocas palabras, los argumentos que se han expuesto conducen a la siguiente conclusión: la reproducción social del capitalismo globalizado se basa en la explotación de recursos energéticos y en la privatización de la función de creación del dinero a través del crédito. Estos dos pilares del desarrollo provocan, sin embargo, importantes riesgos sistémicos, uno a través de la contaminación y del cambio climático, otro a causa de la aparición de burbujas financieras y del exceso de deuda pública y privada. Las crisis económicas y ambientales que amenazan a la sociedad no son eventos que coincidan de forma ocasional, sino fenómenos estructurales e interconectados ${ }^{32}$ que surgen del funcionamiento paradójico y de las contradicciones de los sistemas sociales. Los mecanismos descritos encuentran un encaje muy constructivo en el marco de la teoría de la constitucionalización de Teubner $^{33}$. En efecto, parafraseando al estudioso

\footnotetext{
${ }^{30}$ KALLIS, G., KERSCHNER, C. y MARTÍNEZ-ALIER, J., "The economics of degrowth", Ecological Economics, vol. 84, 2012, p. 172.

${ }^{31}$ Vid. LEGGET, G., The Energy of Nations. Risk blindness and the road to renaissance, Routledge, 2014; vid. también Carbon Tracker Initiative, Unburnable Carbon. Are the world's financial markets carrying a carbon bubble?, noviembre de 2011. Disponible en: http://www.carbontracker.org/wpcontent/uploads/2014/09/Unburnable-Carbon-Full-rev2-1.pdf

32 Desde una perspectiva diferente que igualmente conduce a similares conclusiones sobre la interconexión entre crisis económica y burbujas del mercado de petróleo, vid. CABALLERO, R. J., FARHI, E. y GOURINCHAS, P.O., "Financial Crash, Commodity Prices, and Global Imbalances", Brookings Papers on Economic Activity, 2008, pp. 1-55.

${ }^{33}$ Vid. TEUBNER, G., Constitutional Fragments. Societal Constitutionalism and Globalization, Oxford University Press, 2012.
} 
alemán, el funcionamiento de los sistemas económicos y energéticos desvela los "mecanismos compulsivos" que son responsables de la degeneración de las operaciones de autorreproducción del sistema hacia consecuencias autodestructivas. Dichas consecuencias dan lugar a tres colisiones distintas ${ }^{34}:$ 1) la colisión del imperativo del crecimiento de un sistema con la integridad de los demás sistemas; 2) la colisión de la aceleración del crecimiento de un sistema con su propia autorreproducción; y 3) la colisión de la racionalidad del conjunto de la sociedad global con su contexto ecológico. Volveremos más adelante sobre esta última colisión, pero ahora debe observarse como la evolución de las tres dinámicas enunciadas puede desembocar en una catástrofe social. Justamente la posibilidad de este resultado destructivo, la aproximación a la catástrofe, representa el "momento constitucional”, según Teubner:

Este es el mensaje del constitucionalismo societario. Un ordenamiento constitucional global tiene que enfrentarse al siguiente problema: ¿cómo pueden ejercerse, en los sistemas funcionalizados de las sociedades avanzadas, presiones externas de una fuerza capaz de inducir, en sus correspondientes procesos internos, autolimitaciones en sus posibilidades de acción? Es un [mensaje] subversivo en la medida en que destruye los excesos de las racionalidades autonomizadas, al tiempo que aprovecha sus dinámicas productivas. Se precisa una "constitucionalización híbrida", en la que participen fuerzas externas procedentes de toda la sociedad, no solo de los instrumentos del poder estatal, sino también las reglas jurídicas, así como los contrapesos de la sociedad civil que derivan de los medios de comunicación, de las protestas y movimientos sociales, de los intelectuales, de las ONGs o de los sindicatos. [...] La difícil tarea de alinear recíprocamente la función de un sistema con la aportación a su ambiente en un nivel suficientemente alto, solo puede tener lugar dentro del propio sistema. [...] No hay otra alternativa que experimentar con la constitucionalización ${ }^{35}$.

Desde esta óptica, los sistemas sociales deben desarrollar una "aproximación ecológica" en sentido amplio; es decir, cada sistema debe autolimitarse para no comprometer la sostenibilidad de la reproducción del resto de sistemas, tratando de interiorizar la racionalidad de aquellos. Las constituciones actúan justamente para limitar las racionalidades expansivas por medio del derecho ${ }^{36}$. La constitucionalización en la época del capitalismo global proyecta contrainstituciones para forzar la autolimitación de los

\footnotetext{
${ }^{34}$ Vid. TEUBNER, G., "Costituzionalismo societario e política del comune", Chignola, S., Il diritto del comune. Crisi della sovranità, proprietà e nuovi poteri costituenti, Ombre Corte, 2012, pp. 53-54.

${ }^{35}$ Ibíd., p. 54-55.

${ }^{36}$ Vid. TEUBNER, Constitutional Fragments..., cit., pp. 12, 75-76.
} 
sistemas sociales expansivos. Los derechos fundamentales de este nuevo constitucionalismo trascienden, pues, la dimensión del individuo y protegen los sistemas sociales de las amenazas de "matrices anónimas no individuales" y de los procesos impersonales de las instituciones, networks y sistemas ${ }^{37}$. En otras palabras, mientras que los derechos fundamentales del constitucionalismo estatal surgieron para proteger al individuo del poder político, los "derechos fundamentales sociales", en la era de la globalización policéntrica, deben proteger la sociedad de los excesos de la economía, de la ciencia, de la tecnología, de la religión, etc. Los procesos de constitucionalización deben salvaguardar la continuidad socioecológica ante las amenazas de las matrices hegemónicas totalizantes, amparando la convivencia de diferentes racionalidades y procesos ecológicos, para evitar la autodestrucción de la sociedad; a tal fin, deben asegurar el carácter democrático de la sociedad no solo a través de los procedimientos formalmente democráticos del sistema político, sino también por medio de la democratización de los distintos ámbitos de la sociedad, como la economía o la energía ${ }^{38}$.

En este trabajo se estudia si las crisis económicas, la crisis de la seguridad energética y la crisis ecológica asociada al antropoceno constituyen un momento constitucional para la Unión de la Energía y si dicha Unión puede considerarse un proceso de constitucionalización en términos de salvaguardia contra las matrices anónimas.

\section{EL TRILEMA DE LA ENERGÍA EN EUROPA}

\section{Las dimensiones de la cuestión energética}

La estrategia de la Unión de la Energía de febrero de 2015 puede entenderse solo si se descifran las relaciones de fuerza y las racionalidades subyacentes al "trilema" de la energía en Europa. Es notorio que la cuestión energética tiene tres dimensiones: la seguridad de suministro, el desarrollo económico y la sostenibilidad ambiental, ya que las operaciones del sistema energético están estrictamente vinculadas a la esfera del poder político, a la económica y a la medioambiental. Cada una de ellas responde a unos objetivos: garantizar el acceso a los recursos y el suministro en un sistema dotado de

\footnotetext{
${ }^{37}$ Vid. TEUBNER, G., "The Anonymous Matrix: Human Rights Violation By Private Transantional Actors", Modern Law Review, vol. 69, 2006, pp. 327-346. Disponible en: http://ssrn.com/abstract $=893106$

${ }^{38}$ TEUBNER, "Costituzionalismo societario...", cit., p. 65.
} 
una red óptimamente operativa y distribuida; lograr crecimiento económico y un mercado competitivo; y proteger la integridad ecológica. La búsqueda de una síntesis funcional a tan complejo acoplamiento entre sistemas se ha llevado a cabo mediante el concepto de desarrollo sostenible, es decir, aquel desarrollo que "asegura las necesidades del presente sin comprometer la capacidad de las futuras generaciones para enfrentarse a sus propias necesidades" 39 . Los sistemas políticos y jurídicos nacionales, así como los de gobernanza regional y global, han integrado ampliamente el desarrollo sostenible como "el patrón conceptual definido en el marco de Naciones Unidas para integrar la necesidad de responder a la crisis ambiental en el sistema de valores propugnados por la organización [...]"40. La UE ha incorporado el desarrollo sostenible como objetivo general y como principio jurídico. Por lo tanto, en el espacio de este discurso tiene relevancia evaluar la relación entre energía y desarrollo sostenible en el marco del derecho de la UE.

Se ha hecho énfasis al principio de este trabajo en la centralidad de la cuestión energética en la fundación de Europa y para su propia existencia como sujeto político y como mercado único. La génesis de la CECA se encuentra en la histórica Declaración Schuman del 9 de mayo de 1950, cuyo autor intelectual fue Jean Monnet ${ }^{41}$. Desde ese momento, a través de una aproximación funcionalista, propia y característica de la construcción europea, la persecución de los grandes objetivos de la paz mundial y de una Europa unida iban a ser logrados gracias a un proceso de integración por pasos y "realizaciones concretas" destinados a "crear una solidaridad de hecho" entre los Estados. La creación de esta unidad de producción proyectaba el amplio objetivo de la unificación económica, pero, al mismo tiempo, alimentaba un discurso mucho más ambicioso a largo plazo:

La puesta en común de las producciones de carbón y de acero garantizará inmediatamente la creación de bases comunes de desarrollo económico, primera etapa de la federación europea $^{42}$.

\footnotetext{
${ }^{39}$ Comisión Mundial del Medio Ambiente y del Desarrollo, Nuestro futuro común, Alianza Editorial, Madrid, 1988, p. 29.

${ }^{40}$ JARIA I MANZANO, J., "El constitucionalismo de la escasez (derechos, justicia y sostenibilidad)", Revista Aranzadi de Derecho Ambiental, núm. 30, 2015, pp. 295-349.

${ }^{41}$ Vid. DUCHENE, F., Jean Monnet: the first statesman of interdependence, Norton, 1994.

${ }^{42}$ Declaración Schuman de 9 de mayo de 1950; sobre el tema, vid. MARISCAL BERASTEGUI, N., "Jean Monnet: Plan Schuman, federación y método", Cuadernos Europeos de Deusto, núm. 22, 2000, p. 157 y ss.
} 
A pesar de que desde los inicios y cada vez con mayor fuerza ${ }^{43}$ el debate en torno al proceso de integración europea haya venido desarrollándose en un discurso nítidamente político-constitucional, la lógica de la integración, expresada por la idea reguladora de una “unión cada vez más estrecha", se ha ejecutado mediante la administrativización del proceso de integración, es decir, como una tarea técnica, puesto que los propósitos de constitucionalización en varias ocasiones han encontrado en los poderes políticos nacionales un obstáculo antes que un apoyo ${ }^{44}$. Esta dinámica ha sido muy evidente en el fracaso del Tratado Constitucional y en la reforma llevada a cabo con el Tratado de Lisboa.

En el caso de la energía, esta consideración es especialmente relevante. Por una parte, la cuestión energética se identificaba, desde el comienzo del proceso de integración, como materia esencial de la "constitución económica" de Europa; por otra, esta misma idea planteaba un desafío al espacio político y jurídico de la modernidad: el Estado ${ }^{45}$. La supranacionalización de la energía, como dinámica intrínseca al proyecto de integración europea, es, en efecto, la manifestación de la creciente quiebra de un espacio homogéneo en el que estaban alineados los conceptos de Estado, soberanía, energía, mercado y derecho. Aún hoy, ante la dimensión global de los problemas energéticos, los Estados miembros de la UE retienen la energía como cuestión soberana. Así lo refleja el artículo 194.2 del Tratado de Funcionamiento de la Unión Europea (TFUE) después del Tratado de Lisboa, que mantiene viva la "paradoja de la soberanía", que se da cuando, a pesar de la escala ultranacional de los retos, los Estados no acceden a transferir poder político en un ámbito crucial para lograr una mayor coordinación internacional ${ }^{46}$.

\footnotetext{
${ }^{43}$ En 1956, el abogado general Lagrange afirmó que el tratado CECA debía ser considerado como la "Carta de la Comunidad"; las sentencias Van Gend en Loos (1963) y Costa (1964) consagraron los principios de supremacía y efecto directo de los tratados constitutivos de la CE; luego la jurisprudencia del TJCE de la década de 1980 reconoció los tratados europeos como carta constitucional básica, especialmente en la Sentencia Les Verts, y aún más después del Tratado de Maastricht de 1992, hasta la histórica cumbre de Bruselas del 17 y 18 de junio de 2004, en la que se aprobó el borrador del Tratado constitucional.

${ }^{44}$ Vid. KJAER, P.F., "Three-dimensional Conflict of Laws in Europe", Zentrum für Europäische Rechspolitik ZERP-Diskussionpapier, 2, 2009, p. 8. Disponible en: http://ssrn.com/abstract=1489939

${ }^{45}$ Sobre el Estado-nación como estructura institucional de la modernidad, vid. JARIA I MANZANO, J., La cuestión ambiental y la transformación de lo público, Tirant lo Blanc, 2011, p. 74 y ss.

${ }^{46}$ McGOWAN, F., "International Regimes for Energy: Finding the Right Level for Policy”, Scrase, I. y Mackerron, G. (eds.), Energy for the Future, Palgrave MacMillan, 2009, p. 21.
} 


\section{Los fundamentos teóricos de la política energética europea}

Desde la perspectiva del proceso de integración, la preocupación y el objetivo inicial de la política energética europea era el mercado y no la energía. La creación del mercado interior de la energía (MIE) ${ }^{47}$ ha sido un triunfo del paradigma neoliberal ${ }^{48}$. Según Helm, el MIE se inspira en una idea muy simple, relacionada con las hipótesis de la teoría económica del mercado eficiente:

There would be one unified European energy market. Energy would flow freely across borders, so that customers would be indifferent as to the source of their supplies, and suppliers would compete across the entire European market for customers, and source their electricity and gas from competing generators and upstream gas providers, including liquefied natural gas (LNG), pipe line supplies and indigenous producers. Such a European market would be much more efficient than the patchwork quilt of national and regional companies. There would be at least three core advantages: competition would drive efficiency; there would be greater diversity of supplies and hence greater security of supply; and a single electricity network would reduce the need for capacity margins, and hence a given level of security of supply would require a significantly reduced installed electricity generation capacity ${ }^{49}$.

Por lo tanto, antes de que entrara en vigor el Tratado de Maastricht en 1992, la cuestión energética fue considerada esencialmente en términos de seguridad y mercado. Mientras que el primer término se consideraba un asunto nacional, el segundo venía siendo progresivamente comunitarizado, especialmente por la acción de la Comisión, que actuaba como "agente integrador" bajo su mandato de market-making empleando las herramientas de la política de competencia, y por la creación de una agencia de los reguladores energéticos ${ }^{50}$. Esta aproximación al problema es ampliamente compartida a nivel global tanto en la práctica como en la teoría, configurando la visión mainstream, tal como explica Proedrou:

[...] Mainstream work remains fundamentally preoccupied with the security, political and economic aspects of energy issues. This derives from a largely unchallenged

\footnotetext{
${ }^{47}$ En virtud de las directivas aprobadas en la mitad de la década de 1990.

${ }^{48}$ GOLdThaU, A. y SITTER, N., The Power of Paradigms: The EU and Global Energy Policy, 17 de enero, 2012. Disponible en: http://ssrn.com/abstract=1986832.

${ }^{49}$ HELM, D., “The European framework for energy and climate policies”, Energy Policy, 64, 2014, p. 30.

${ }^{50}$ Vid. PAKALKAITE, V., Competence Creep Through the Backdoor: EU Energy Regulation and Competition Policy, 5th Biennial ECPR Standing Group for Regulatory Governance Conference, Barcelona, 25-27 de junio de 2014, pp. 1-2.
} 
assumption that our societies have to grow infinitely. Since energy is the backbone of all societies - and the history of humanity can also be read as the history of energy production and consumption that has allowed the human civilization to achieve goals that were regarded unimaginable in the past - it is considered given that this is the path all societies should take: strive hard to produce more energy in order to increase technological innovations that could substantially boost their welfare level. The quest for energy, in this context, is considered a sine qua non for all, and those countries that are able to master the most resources are the ones to progress further than others, and will be in position to increase their welfare and perhaps also dominate others. Energy issues, thus, are severely economized and securitized ${ }^{51}$.

La firma del Tratado de Maastricht añadió el concepto de "crecimiento sostenible que respete el medio ambiente" a las obligaciones de la Comunidad Europea, así como el principio de precaución en el artículo en el que se basaba la política ambiental (art. 130 R). En el Consejo Europeo de Goteburgo de junio 2001, los líderes europeos lanzaron la primera Estrategia de Desarrollo Sostenible (EDS), en la que se priorizaba la necesidad de "limitar el cambio climático e incrementar el uso de energías limpias". La EDS pedía un nuevo enfoque para la formulación de políticas en las que el desarrollo sostenible se colocara en el centro de todas las políticas. La intención era introducir una perspectiva coherente y a largo plazo que sustituyera la aproximación hasta entonces vigente, que se había centrado demasiado en los costos a corto plazo $^{52}$. El Consejo Europeo de junio de 2006 adoptó una revisión de la EDS que identificó como objetivos principales la necesidad de salvaguardar la capacidad de carga de la Tierra, proteger los recursos naturales del planeta y mejorar la calidad del medio ambiente. En 2009, en el apogeo de la crisis económica mundial, la EDS se actualizó otra vez ${ }^{53}$. En definitiva, la UE ha incorporado la sostenibilidad en muchos de sus ámbitos de actuación. En diciembre de 2008, los legisladores de la UE acordaron un paquete sobre clima y energía que estableció objetivos vinculantes para la Unión en $2020^{54}$. Entre 1996 y 2009 se

\footnotetext{
${ }^{51}$ PROEDROU, F., "Rethinking energy security: An inter-paradigmatic debate", Policy Paper, Hellenic Foundation for European \& Foreign Policy, núm. 24, febrero de 2015, p. 5.

${ }^{52}$ COM(2001)0264 final, A Sustainable Europe for a Better World: A European Union Strategy for Sustainable Development.

${ }^{53} \mathrm{COM}(2009) 0400$ final, Mainstreaming sustainable development into EU policies: 2009 Review of the European Union Strategy for Sustainable Development.

${ }^{54}$ Vid. COM(2008)30 final, Dos veces 20 para el 2020. El cambio climático, una oportunidad para Europa; Consejo Europeo, Paquete sobre Clima y Energía. Elementos del compromiso final acordados por el Consejo Europeo del 12 de diciembre de 2008. Disponible en: http://www.consilium.europa.eu/uedocs/cmsUpload/st17215.en08.pdf. Las medidas legislativas
} 
adoptaron tres paquetes de medidas legislativas con el fin de armonizar el mercado interior de la energía ${ }^{55}$. En el marco estratégico para la energía y el clima para el período 2020-2030, aprobado en enero de $2014^{56}$ y alineado con la hoja de ruta de la energía para el $2050^{57}$, la Comisión propone un nuevo objetivo de reducción de las emisiones internas de gases de efecto invernadero del 40\% con respecto al 1990, al tiempo que el objetivo general europeo para las energías renovables será como mínimo del 27\%. Según la Comisión, la UE debería continuar por "la senda menos costosa hacia la economía hipocarbónica [y] todo lo posible orientada hacia al mercado" ${ }^{\text {. }}$. La novedad más destacable del marco estratégico 2020-2030 es que el nuevo objetivo de energía renovable será vinculante para la UE, si bien esto no se traducirá en objetivos nacionales vinculantes a través de la legislación de la UE. Este abandono de los objetivos vinculantes en aras de la flexibilidad nacional implica que serán los Estados los que tendrán que autocomprometerse para lograr de forma colectiva el objetivo de la UE. A tal fin, se articulará un nuevo proceso iterativo de gobernanza que requerirá una actividad de coordinación de los planes nacionales y una apuesta por un enfoque cooperativo. La cooperación aparece como la característica más distintiva de la administración europea integrada del marco de la energía y el clima, dirigida a salvar las preferencias nacionales siempre que sean compatibles con una mayor integración de los mercados y el aumento de la competencia. Estos desarrollos de la política energética validan la tesis de la naturaleza híbrida de la UE elaborada por Kjaer, según la cual la UE opera entre los Estados miembros y las estructuras globales transnacionales como

adoptadas fueron las siguientes: Directiva 2009/29/EC, Decisión 406/2009/EC, Directiva 2009/28/EC y Directiva 2009/31/EC

${ }^{55}$ El primer paquete legislativo (la Directiva 96/92/CE sobre normas comunes para el mercado interior de la electricidad y la Directiva 98/30/CE sobre normas comunes para el mercado interior del gas natural) fue sustituido en 2003 por un segundo paquete legislativo que permitió la entrada de nuevos proveedores de gas y electricidad a los mercados de los Estados miembros, y abrió la posibilidad de que los consumidores (los industriales desde el 1 de julio de 2004 y los domésticos desde el 1 de julio de 2007) pudieran elegir libremente a su proveedor de gas y electricidad. En abril de 2009 se aprobó un tercer paquete legislativo con el que se perseguía introducir un mayor grado de liberalización en el mercado interior de la electricidad y el gas y que introducía modificaciones en el segundo paquete: la Directiva sobre la electricidad (2009/72/CE), por la que se deroga la Directiva 2003/54/CE, y la Directiva sobre el gas (2009/73/CE), por la que se deroga la Directiva 2003/55/CE.

${ }^{56}$ Vid. COM(2014) 15 final.

${ }^{57}$ Vid. COM(2011)0885 final. La hoja de ruta reitera el compromiso de reducción de los gases de efecto invernadero entre un $80 \%$ y un $95 \%$ por debajo de los niveles de 1990 para 2050, tal como se acordó en el Consejo Europeo de octubre de 2009.

${ }^{58} \operatorname{COM}(2014) 15$ final, pp. 6-7. 
un ordenamiento ni completamente jerárquico como los Estados nacionales ni radicalmente heterárquico como las estructuras jurídicas globales:

From an overall perspective, the EU can nonetheless be understood as resting on a twodimensional organizational structure in the sense that it, on the one hand, contains a hierarchical governing dimension, consisting of the triangular relationship between the Council of the European Union (the Council), the Commission of the European Communities (the Commission) and the European Parliament (EP) and a heterarchical dimension consisting of a multitude of governance structures (GS), such as the Open Method of Co-ordination (OMC), Comitology and (regulatory) agencies. The first dimension constitutes the political system of the EU. The latter dimension, on the other hand, represents forms through which the political system of the EU ensures its embeddedness in its environment. This dimension therefore reflects the functionally differentiated basic structures (Tiefenstrukturen) of the wider society just as it is directly aimed at overcoming the distinction between the public and the private spheres of society ${ }^{59}$.

Desde esta perspectiva es posible interrogarse sobre cómo están siendo constitucionalidazas las relaciones entre las dimensiones del governing y de la governance, y entre las diferentes estructuras de governance ${ }^{60}$. La aplicación de este enfoque de análisis brinda interesantes consideraciones respecto a la cuestión energética, ya que permite captar las tensiones verticales entre la UE y los Estados miembros, así como las horizontales, por una parte, entre los diferentes Estados y, por otra, entre sistemas sociales.

\section{La integración energética europea entre mercado y desarrollo sostenible}

En primer lugar, a pesar de los esfuerzos de integración, los beneficios que hubiera tenido que reportar la construcción del MIE no han sido alicientes suficientes para la cooperación. Asimismo, aunque el establecimiento de los primeros objetivos en materia de clima y energía ha sido un logro de la UE, su implementación se ha visto socavado por las preocupaciones en materia de competitividad, de tal manera que el poder normativo de la UE no ha sido correspondido con la necesaria cooperación entre

\footnotetext{
${ }^{59}$ KJAER, "Three-dimensional...", cit., pp. 1 y 2.

${ }^{60}$ Ibíd., p. 2.
} 
Estados Miembros para alcanzar los objetivos fijados. Por lo tanto, no se ha culminado la realización del MIE prevista para finales de 2014. El motivo por el que no se ha logrado una unión más estrecha en materia de energía, según los analistas, reside en que el incentivo a la cooperación más poderoso es el reconocimiento de la vulnerabilidad energética, es decir, la dimensión relativa a la seguridad energética ${ }^{61}$. A pesar de los esfuerzos en materia de seguridad del suministro de electricidad, gas natural y petróleo $^{62}$, este aspecto aún está en mano de los Estados, los cuales, amparándose en su soberanía e intereses geoestratégicos, acostumbran a abordar este problema en clave nacional y negociando acuerdos bilaterales con los suministradores externos a la UE. Este tema se ha evidenciado con toda claridad en la reciente Estrategia Europea de la Seguridad Energética:

[1]a UE continúa siendo vulnerable a las perturbaciones energéticas externas [...]. La UE necesita, por tanto, una estrategia realista de seguridad energética que refuerce la capacidad de resistencia frente a estas perturbaciones e interrupciones del abastecimiento a corto plazo y reduzca la dependencia respecto a determinados combustibles, suministradores y rutas de suministro a largo plazo. Los responsables de la elaboración de las políticas nacionales y de la Unión deben exponer claramente a los ciudadanos las repercusiones de esta reducción de la dependencia. [...] No obstante, con demasiada frecuencia los problemas de seguridad energética se abordan únicamente a escala nacional, sin tener plenamente en cuenta la interdependencia entre los Estados miembros. La clave para la mejora de la seguridad energética es, en primer lugar, un planteamiento más colectivo mediante un mercado interior eficaz y una mayor cooperación en los

\footnotetext{
${ }^{61}$ Vid. HEDBERG, A., "EU's quest for energy security. What role for Energy Union?", European Policy Centre, Policy Brief, 3 de marzo de 2015. Disponible en: http://www.epc.eu/documents/uploads/pub_5374_eu_s_quest_for_energy_security.pdf

62 "La Directiva 2005/89/CE establece un conjunto de medidas dirigidas a salvaguardar la seguridad del suministro de electricidad, con objeto de garantizar el adecuado funcionamiento del mercado interior de la electricidad, un nivel apropiado de interconexión entre los Estados miembros y de capacidad de generación, así como el equilibrio entre la oferta y la demanda. A la vista de la crucial importancia del gas para el suministro energético de la Unión Europea, y como respuesta a la crisis del gas entre Rusia y Ucrania durante el invierno de 2008/2009, en 2010 se adoptó el Reglamento (UE) no 994/2010 sobre medidas para garantizar la seguridad del suministro de gas. Dicho Reglamento tiene por objeto fortalecer los mecanismos de prevención y respuesta ante las crisis. A fin de asegurar el suministro de petróleo, la Directiva 2009/119/CE obliga a los Estados miembros a mantener unas reservas mínimas de petróleo equivalentes a las importaciones netas medias diarias de 90 días o a 61 días de consumo interno medio diario, en función de la cantidad que resulte mayor", KEREBEL, C., "El mercado interior de la energía", Fichas técnicas sobre la Unión Europea, 3/2015. Disponible en: http://www.europarl.europa.eu/aboutparliament/es/displayFtu.html?ftuId=FTU_5.7.2.html
} 
ámbitos regional y europeo, en particular para coordinar los desarrollos de redes y abrir mercados $^{63}$.

Por lo tanto, la hasta ahora fallida constitucionalización europea de la energía se deriva de la insuficiente síntesis entre la dinámica de integración del MIE, mayormente perseguida a nivel de las estructuras de la UE, y la dimensión de la seguridad, vinculada al principio de soberanía permanente sobre los recursos energéticos radicado a nivel de los Estados nacionales. En otras palabras, los Estados perseveran en la utopía constitucional de poder articular, a partir de su soberanía territorial, un espacio de intercambio y explotación seguro ${ }^{64}$ para la energía, mientras que el sistema energético ha trascendido los límites nacionales y depende de las estructuras europeas y globales de poder para que se garantice el flujo de recursos energéticos y la inversión de capitales necesarios para su explotación, al tiempo que la afectación ecológica, especialmente en términos de emisiones de gases de efecto invernadero, es una vertiente obviamente transnacional.

La referida dimensión global de la gobernanza energética ${ }^{65}$ resultante de la emancipación del sistema energético del espacio estatal implica que el derecho transnacional ya no puede depender del derecho producido por los sistemas políticos nacionales, sino que debe generar nuevos principios jurídicos a partir de las relaciones con otras estructuras y sistemas que se encuentran fuera del espacio de los Estados. Mientras que el constitucionalismo de la modernidad se fundamentaba en el acoplamiento entre derecho y política con el fin de establecer límites al poder político, el nuevo constitucionalismo pluralista y transnacional se caracteriza por establecer acoplamientos que tienen un valor constitucional con otros sistemas ${ }^{66}$ como el económico, el científico, el energético, etc. Los problemas que el nuevo constitucionalismo debe afrontar son los excesos de los poderes económicos,

\footnotetext{
${ }^{63} \operatorname{COM}(2014) 330$ final, Estrategia Europea de la Seguridad Energética, del 28/05/2014.

${ }^{64}$ Parafraseando a JARIA I MANZANO, J., "Crisis ambiental y decisión política: la fragmentación de los espacios políticos", ponencia presentada en el XI Congreso AECPA, 2013, p. 2. Disponible en: http://www.aecpa.es/uploads/files/modules/congress/11/papers/980.pdf

${ }^{65}$ Sobre la temática de la gobernanza global energética, vid. DUBASH, N.K. y FLORINI, A., "Introduction to the Special Issue: Governing Energy in a Fragmented World", Global Policy, vol. 2, special issue, septiembre 2011, pp. 1-5; DUBASH, N.K. y FLORINI, A., "Mapping Global Energy Governance", Global Policy, vol. 2, special issue, septiembre 2011, pp. 6-18; SOVACOOL, B.K. y FLORINI, A. "Examining the Complications of Global Energy Governance", Journal of Energy \& Natural Resources Law, vol. 30, núm. 2, 2012, pp. 235-264.
}

${ }^{66}$ Vid. KJAER, “Three-dimensional..., cit., p. 5. 
energéticos, etc., en el marco del capitalismo avanzado. Es un constitucionalismo que tiene efectos horizontales en la medida en que debe proteger las esferas sociales de la expansión de otras racionalidades sociales que pueden colonizar la sociedad. Por lo tanto, el constitucionalismo posmoderno debe establecer nuevos límites a las amenazas procedentes de nuevas matrices sociales y abrirse cognitivamente a las racionalidades de otros sistemas sociales y comunidades. Según Prandini:

Quando la differenziazione funzionale della società-mondo decostruisce le categorie moderne del diritto e quando la gerarchia delle fonti lascia spazio a una infinita rete eterarchica di rimandi giuridici, emerge il problema della "costituzione" del nuovo arcipelago globale. $\mathrm{O}$ almeno così la faccenda appare a chi abbia a cuore la possibilità stessa del diritto e della giustizia nell'epoca della globalizzazione ${ }^{67}$.

De acuerdo con Teubner, la justicia es un concepto relacional que debe elaborarse desde el interior de cada subsistema social, al tiempo que el derecho se enfrenta al desafío de la justicia no solo mediante el control de la consistencia interna del propio derecho (coherencia/incoherencia de las decisiones y del discurso jurídico), sino también mediante la reconstrucción de la racionalidad de los otros discursos interesados y la persecución de fines de justicia material. Por este motivo, tiene sentido hablar de justicia energética y constitucionalismo de la energía ${ }^{68}$. Sin embargo, contrariamente a lo que sería necesario, tanto a nivel internacional como a nivel europeo se ha producido una colonización de los sistemas sociales por parte del sistema económico y de su racionalidad; en otras palabras, el discurso económico neoliberal ha logrado una forma específica de gubernamentalidad $^{69}$. En palabras de Kessler, Leander y Van Munster:

[Neoliberalism] can be characterised as the entry of the market in politics: techniques on the basis of market principles, instrumental rationality, benchmarking, statistics, and risk based categorisations. Risk fosters exactly this neoliberal governmentality, as Leander and van Munster nicely summarised: "Neo-liberal governmentality situates human activity within an economic calculus. It relocates activities from the public realm to the market by means of direct privatization, public-private partnerships, outsources, the

\footnotetext{
${ }^{67}$ Vid. PRANDINI, "La costituzione del diritto...", cit., pp. 192-193.

${ }^{68}$ Ibíd., p. 205.

${ }^{69}$ Sobre la diferencia entre las nociones de gobernanza y gubernamentalidad, LÖVBRAND et ál. consideran lo siguiente: "[...] while the governance concept is concerned with the loci and modes of governing, the governmentality concept draws attention to the systematic thinking that renders different governing strategies possible". LÖVBRAND, E., STRIPPLE, J. y WIMAN, B., "Earth System governmentality: Reflections on science in the Anthropocene", Global Environmental Change, vol. 19, núm. 1, 2009, pp 7-13.
} 
creation of new markets, the making of internal markets and the establishment of conditions for competitive success" ${ }^{, 70}$.

A nivel internacional, explica Jaria, el concepto de desarrollo sostenible ha hecho posible la incorporación de la crisis ambiental en el discurso hegemónico, "sin incidir en la estructura institucional del capitalismo global y su favorecimiento de la desregulación creciente en materia de comercio e inversiones"71. A nivel de la UE, ya hemos observado como el paradigma neoliberal constituye la base teórica sobre la cual se ha construido el MIE. Desde la década de 1990, y si se quisiera determinar con más detalle un momento de salida este podría identificarse con la Directiva 90/547/CE, la Comunidad Europea pasa de una política de intenciones a una basada en principios y reglas $^{72}$. En un estudio sistemático sobre los principios de derecho europeo de la energía, Parente ha explicado que en dicho ámbito existen dos macroprincipios, que “constituyendo preceptos de optimización del ordenamiento, permiten enmarcar cualquier manifestación según líneas y criterios predeterminados" ${ }^{73}$; dichos macroprincipios son: 1) el principio de buen funcionamiento del mercado interior de la energía; y 2) el principio de seguridad en el abasteciemiento. En cuanto al primero, "para que pueda afirmarse el respeto de este principio, es necesario que se satisfagan dos condiciones: 1) que se cree un mercado interior de la energía; y 2) que se asegure su correcto funcionamiento" ${ }^{74}$. A tal fin, el derecho europeo ha empleado todas las herramientas de liberalización y de regulación pro competencia, y para el funcionamiento del mercado se ha acudido a los principios de common carrier, de unbundling, de no discriminación, de reciprocidad, de confidencialidad, de transparencia y a la doctrina de las essential facilities. Por lo que se refiere al principio de seguridad en el abastecimiento, este debería entenderse como "un mandato de optimización del ordenamiento que, en presencia de una red perfectamente operativa y eficazmente distribuida, permite - en todo momento- garantizar un equilibrio

\footnotetext{
${ }^{70}$ KESSLER, O., "The same as it never was? Uncertainty and the changing contours of international law", Review of International Studies, 37, 2011, p. 2171, en el que se cita a LEANDER, A. y Van MUNSTER, R., "Private Security Contractors in the Debate about Darfur: Reflecting and Reinforcing Neo-Liberal Governmentality", International Relations, vol. 21, núm. 2, junio de 2007, pp. 201-216.

${ }^{71}$ JARIA I MANZANO, "El constitucionalismo de la escasez...", cit.

${ }^{72}$ PARENTE, A., Principios de Derecho de la Energía, Aranzadi, 2010, p. 35 y ss.

${ }^{73}$ Ibíd., p. 32 y ss.

${ }^{74}$ Ibíd., p. 286.
} 
deseable entre oferta y demanda energética"75. Por lo que en esta vertiente se aplicarían los instrumentos de intervención en la oferta y en la demanda. Es interesante resaltar como la doctrina citada considera que en el ordenamiento jurídico europeo existirían objetivos de garantía y objetivos de promoción. A los primeros pertenecen el mercado interior y la seguridad de abastecimiento energético, que, al incorporar un fin de garantía, conllevarían una concesión general de poderes a la Unión para su definición y control o para llevar a cabo las innovaciones institucionales necesarias. En cambio, los objetivos de promoción, que abarcarían el ahorro y la eficiencia energéticos, el desarrollo de energías renovables y las interconexiones de redes, implican para la Unión un rol distinto, un papel complementario de los Estados miembros o, en cualquier caso, de indirizzo político. Cabe insistir en que las facultades de actuación serían distintas, ya que en el primer supuesto correspondería a la UE una posición de dominio, mientras que, en el segundo supuesto, una posición de promoción con facultades menos incisivas $^{76}$. Continúa Parente argumentando que la expresión "atendiendo a la necesidad de preservar el medio ambiente" contenida en el artículo 194.1 TFUE, sobre política energética, se configuraría como "un elemento condicionante de toda decisión asumida en el sector energético. [...] Esto no quiere decir que la política energética sea un modus de la política ambiental, sino sólo que ésta última la condiciona",77. En realidad, según este autor, el artículo 194.1 debería interpretarse en el sentido de que existe "una obligación de una previa evaluación y no la obligación de una consonancia entre la evaluación realizada y la decisión asumida: en otras palabras, hay que tomar en consideración los objetivos de tutela ambiental, pero las evaluaciones realizadas no serían necesariamente vinculantes" ${ }^{, 78}$.

Esta interpretación doctrinal pone de manifiesto que el derecho no ha podido incorporar suficientemente el discurso ambiental, mientras que sus principios han sido profundamente colonizados por el discurso del mercado eficiente y operativamente capaz de satisfacer demanda y oferta de energía. Morata y Solorio han evidenciado como en el discurso que sustenta la sostenibilidad ambiental de la política energética se ha producido gradualmente un cambio: mientras que en el período entre 1998 y 2005 ,

\footnotetext{
${ }^{75}$ PARENTE, Principios..., cit., p. 290.

${ }^{76}$ Ibíd., pp. 49-50.

${ }^{77}$ Ibíd., p. 45.

${ }^{78}$ Ibíd., p. 45.
} 
especialmente en virtud del Tratado de Ámsterdam, el foco estaba centrado en la sostenibilidad y en el principio de integración ambiental, desde el 2005 el discurso ha ido centrándose en el cambio climático, lo que ha permitido variar el discurso de conseguir un sistema energético sostenible a introducir una visión gerencial y tecnológica del cambio climático en términos de reducción de las emisiones de $\mathrm{CO}_{2}{ }^{79}$. De ahí que el fomento de la captura y el almacenamiento de carbono ${ }^{80}$ como remedio a la explotación de recursos fósiles, la explotación del gas natural convencional o no convencional, que emite menos $\mathrm{CO}_{2}$ que otros combustibles fósiles, la energía nuclear limpia, etc., se transforman paradójicamente en opciones viables para un sistema energético más verde ${ }^{81}$. De esta manera, se ha producido lo que Stevenson denomina la “institucionalización de la insostenibilidad", dado que:

The specific sources of emissions, as well as the social and political objectives they serve, are treated as irrelevant: avoiding dangerous climate change simply requires limiting overall global emissions. Global climate governance has thereby become a task principally of mitigating greenhouse gas emissions at the cheapest possible source, rather than one of transforming the political, economic, and cultural drivers of excessive emissions ${ }^{82}$.

La sostenibilidad ambiental debería transformarse en un objetivo consustancial al proyecto de integración europea ${ }^{83}$. De este modo, se introducirían los límites necesarios a la expansión compulsiva de los sistemas que amenazan la reproducción social en el sistema ecológico. En lo que se refiere al derecho de la UE, el desarrollo sostenible es a

\footnotetext{
${ }^{79}$ MORATA, F. y SOLORIO SANDOVAL, I., "When 'green' is not always sustainable: the inconvenient truth of the EU energy policy”, Carbon Management, 4, 5, 2013, pp. 555-563.

${ }^{80}$ Sobre la introducción del CCS en el discurso hegemónico de las políticas de la UE, vid. MARTÍNEZ ARRANZ, A., "Carbon capture and storage: Frames and blind spots", Energy Policy, vol. 82, julio de 2015, pp. 249-259.

${ }^{81}$ Según MORATA y SOLORIO: "Against this background, what we are witnessing is the (apparently successful) attempt of traditional sources of energy to 'greenwash' their reputation under the guise of climate change. [...] The 'inconvenient truth' of EU energy policy is that, in its effort to fight against climate change in a strict sense, it is disregarding sustainable development as a more comprehensive environmental concern. Thus, rather than building a truly sustainable energy system based on renewable power, it is progressively opening the door to unsustainable energy sources [...]", vid. MORATA y SOLORIO SANDOVAL, "When 'green'...", cit., p. 561.

${ }^{82}$ STEVENSON, H., Institutionalizing Unsustainability: The Paradox of Global Climate Governance, University of California Press, 2013.

${ }^{83}$ Sobre la existencia de la obligación de adoptar medidas de tal manera que el desarrollo sostenible se transforme en el fin último de la integración europea, vid. SJÅFELL, B., "Sustainable Development, EU Law and Companies: The EU Law Framework for the Sustainable Companies Project", International and Comparative Corporate Law Journal, vol. 8, núm. 1, 2011, pp. 1-14. Disponible en: http://papers.ssrn.com/sol3/papers.cfm?abstract_id=1712789
} 
la vez un objetivo general y un principio de derecho. Desde el Tratado de Lisboa, el desarrollo sostenible ha aumentado su "peso específico" normativo. El artículo 3 del Tratado de la Unión Europea (TUE) establece que el desarrollo sostenible global es un objetivo general de la Unión. Por otro lado, la posición del desarrollo sostenible y la de la protección del medio ambiente se han visto reforzadas tras la codificación del principio de desarrollo sostenible en el "principio de la integración ambiental" del artículo 11 TFUE:

Las exigencias de la protección del medio ambiente deberán integrarse en la definición y en la realización de las políticas y acciones de la Unión, en particular con objeto de fomentar un desarrollo sostenible.

Según Sjåfjell, la base jurídica del artículo 11 es aplicable sin ninguna exclusión a todas las actividades y políticas de la UE y, además, el principio de integración contiene una regla de priorización a favor de la sostenibilidad: "Economic development has traditionally had a strong position, and social development considerations (at least in Europe) have their advocates in a way that protection of the environment does not yet have" 84 . Por lo tanto, dado que de los objetivos generales derivan obligaciones para las instituciones de la UE, "article 11 strengthens the position of sustainable development as a general objective, and it establishes the direction to be followed when integrating environmental protection requirements into all sectors" ${ }^{~} 85$. En la medida en que el desarrollo sostenible es un principio que requiere un ejercicio de ponderación con las otras dimensiones del desarrollo, se debería argumentar que "this balancing must have sustainable development as its ultimate goal, with emphasis given to its environmental protection dimensión" $" 86$. Sin embargo, tal como venimos observando, la síntesis de la racionalidad ecológica que implicaría internalizar la sostenibilidad como límite a la expansión del sistema energético no tiene lugar en el marco de la UE, ya que el desarrollo sostenible va acompañado de una priorización de la lógica económica y tecnológica ${ }^{87}$. La intervención europea en la política energética continúa orientándose hacia la consecución del MIE como estructura fundamental para garantizar la seguridad

\footnotetext{
${ }^{84}$ SJÅFELL, "Sustainable Development...", cit., p. 8.

${ }^{85}$ Ibíd., p. 9.

${ }^{86}$ Ibíd., p. 10.

${ }^{87}$ Ibíd., p. 13.
} 
del suministro y para abordar los desafíos del cambio climático ${ }^{88}$. Después de tres paquetes legislativos y de la reforma del Tratado de Lisboa, que ha añadido la competencia compartida entre la UE y los Estados en materia de energía, la construcción del MIE no se ha logrado aún y el principio de soberanía nacional guía las decisiones sobre el mix energético de cada nación. Las medidas legislativas adoptadas por el procedimiento ordinario, de acuerdo con el artículo 194.2 del TFUE, "no afectarán al derecho de un Estado miembro a determinar las condiciones de explotación de sus recursos energéticos, sus posibilidades de elegir entre distintas fuentes de energía y la estructura general de su abastecimiento energético". Estos son los antecedentes del nuevo paquete sobre la Unión de la Energía, lanzado en febrero de $2015^{89}$.

\section{La europeización de la seguridad y la Unión de la Energía como dispositivo}

La nueva estrategia de la Unión de la Energía es un programa cuyo fin es elevar a la dimensión europea la cuestión de la seguridad y utilizarla como palanca para la integración, vistos los resultados insatisfactorios del vector mercado y del vector ambiental para lograr una integración más estrecha. En este sentido, el discurso desarrollado en la $\operatorname{COM}(2015) 80$ es muy claro:

Aspiramos a una Unión de la Energía en la que los Estados miembros se den cuenta de que dependen unos de otros para garantizar a sus ciudadanos una energía segura, sobre la base de una solidaridad y confianza auténticas, que hable con una sola voz en asuntos de alcance mundial $^{90}$.

Por lo tanto, se trata de rescatar, del principio de soberanía nacional, la seguridad energética e introducirla en el circuito híbrido del sistema europeo; de esta manera, se darían las condiciones para la síntesis funcional entre mercado, desarrollo sostenible y seguridad que el desajuste estructural entre la UE y los Estados miembros no ha logrado hasta la fecha. Debe decirse también que la Comunicación sobre la Unión de la Energía es desde el propio punto de vista del discurso una hibridación. En efecto, su base es el "manifiesto" del entonces primer ministro de Polonia y ahora presidente del Consejo

\footnotetext{
${ }^{88}$ PÉREZ DE LAS HERAS, B., "Las políticas de seguridad energética en la Unión Europea y los Estados Unidos: desafíos globales y compromisos comunes en la transición hacia un modelo energético más sostenible", Revista de Derecho Comunitario Europeo, núm. 47, 2014, p. 20.

${ }^{89}$ Toda la información puede consultarse en la página web sobre la Unión de la Energía: http://ec.europa.eu/priorities/energy-union/index_en.htm.

${ }^{90} \operatorname{COM}(2015) 80$ final.
} 
Europeo, Donal Tusk, "A united Europe can end Russia’s energy stranglehold" "91, cohonestado con el discurso de la política energética de la UE. La propuesta original de Tusk fue impulsada por los sucesos relacionados con Ucrania, Rusia y las repetidas crisis del gas sobre la base de una asunción: la excesiva dependencia de Rusia debilita a Europa:

The EU is creating a banking union, a Europe-wide underpinning for its financial institutions. [...] I therefore propose an energy union. It will return the European project to its roots. [...] Whether in coal, steel, uranium, credit or gas, the principal idea of the EU has always been to bring Europe together, deepening our security and establishing fair rules where the free market is lacking. An energy union, too, would be based on solidarity and common economic interests ${ }^{92}$.

Los seis pilares que, según Tusk, sustentarían la Unión de la Energía son: 1) la creación de un mecanismo de negociación y compra común europeo del gas ruso para así enfrentar al suministrador dominante un sujeto dominante en la demanda; 2) la habilitación de instrumentos para hacer efectiva la solidaridad entre los Estados en caso de interrupción del suministro; 3) la construcción de las infraestructuras necesarias para permitir las interconexiones y el almacenamiento de energía y así reducir la exposición de los países cuyo abastecimiento depende de un único operador como Gazprom; 4) el uso extensivo de los recursos fósiles domésticos, incluyendo el shale gas y el carbón; 5) la promoción de la diversificación del suministro acudiendo al mercado global de la energía, especialmente del gas; $y$, finalmente, 6) la potenciación de la Comunidad de la Energía, organización internacional creada por el Tratado de Atenas de 2005 para unir la UE y los países del sudeste de Europa y la región del mar Negro.

Resulta patente, pues, que en semejante visión de la Unión de la Energía no solo la sostenibilidad ecológica y la protección ambiental no se contemplan, sino que explícitamente se predica la reproducción del sistema energético y su aseguramiento a través del uso de combustibles fósiles: "In the EU's eastern states, Poland among them, coal is synonymous with energy security"93. La viabilidad de este proyecto de integración energética de la Unión precisaba, sin embargo, ser absorbido por el discurso

\footnotetext{
${ }^{91}$ La visión de la Unión de la Energía de Tusk fue publicada en el Financial Times el 21 de abril de 2014. Disponible en: http://www.ft.com/intl/cms/s/0/91508464-c661-11e3-ba0e-00144feabdc0.html\#ft-articlecomments

${ }^{92}$ TUSK, D., “A united Europe can end Russia's energy stranglehold”, Financial Times, 21 de abril, 2014.

${ }^{93}$ Ibíd., “A united Europe...," cit.
} 
generado en el marco de la política energética europea desarrollada a lo largo de los años $^{94}$.

La nueva estrategia de la Unión de la Energía, tal como se expone en la COM(2015)80, debería establecer las herramientas necesarias para hacer frente a las perturbaciones en el suministro de energía derivadas de eventos geopolíticos a los que está expuesta la UE a causa de su dependencia energética del petróleo y del gas exterior" ${ }^{95}$. El "market approach" del tercer paquete de energía persiste y se añade la necesidad de construir las infraestructuras físicas necesarias para el mercado. Para conseguir este propósito, sería necesaria una política energética coherente y unitaria que supere la situación de impasse en la cual se encuentra la UE. La Unión todavía no habla con una sola voz ni dispone de un verdadero mercado interior; al contrario, persiste en su seno una fragmentación de veintiocho mercados nacionales soberanos de la energía. Ante este panorama, las instituciones de la UE quieren avanzar hacia la construcción de un mercado integrado, competitivo y cuyo buen funcionamiento asegure precios asequibles para sus usuarios. Al mismo tiempo, la UE está comprometida con el desarrollo de una política energética coherente con el desafío del cambio climático y con el objetivo de dirigir su transición energética hacia un modelo más eficiente e hipocarbónico. De esta manera conseguiría también proyectar su poder normativo a nivel internacional, también con vistas al esperado acuerdo sobre clima que deberá adoptarse en París en diciembre 2015. En realidad, la realización de los objetivos relativos a las emisiones y las energías renovables y la eficiencia energética, más que estar vinculada a la sostenibilidad como fin último, parece un instrumento para contribuir a la seguridad energética, tema que es el verdadero driver de la estrategia europea ${ }^{96}$. La estrategia responsabiliza de la situación de crisis a los Estados y su propósito manifiesto es fracturar la protección soberana de la energía. La energía debe fluir libremente en el mercado y los territorios nacionales ya no son los espacios de intercambio seguro garantizados por el derecho. En la nueva estrategia de la energía la solidaridad y los enfoques conjuntos ya no se predican entre Estados, sino entre regiones:

\footnotetext{
${ }^{94}$ Sobre la evolución de la política energética europea, vid. TALUS, K., EU Energy Law and Policy. A Critical Account, Oxford University Press, 2013.

${ }^{95}$ La UE constituye el mayor importador de energía del mundo: según datos relativos al 2012, la UE(28) depende en un 53,4\% de las importaciones energéticas exteriores; en particular, el 65,8\% del gas y el $86,4 \%$ del petróleo consumidos en la UE deben importarse de terceros países. Vid. Comisión Europea, Energy statistical pocketbook, Publications Office of the European Union, 2014, p. 24. Disponible en: http://ec.europa.eu/energy/sites/ener/files/documents/2014_pocketbook.pdf

${ }^{96}$ Vid. HEDBERG, "EU's quest...", cit.
} 
En una Unión de la Energía, los Estados miembros deben coordinarse y cooperar con sus vecinos para elaborar sus políticas de energía. La ejecución técnica de los diferentes elementos de la estrategia de la Unión de la Energía será muy compleja. Algunos elementos, como las nuevas disposiciones de mercado para los mercados a corto plazo del gas y la electricidad o la integración de las actividades de los gestores de redes de transporte, deben desarrollarse y aplicarse a nivel regional, como un paso hacia la plena integración del mercado a nivel de la UE. [...] La Comisión se asegurará de que todas las iniciativas regionales evolucionen de manera coherente y den lugar a un mercado único de la energía plenamente integrado ${ }^{97}$.

El "concepto fluido" de Unión de la Energía 98 tiene como propósito poner bajo un mismo paraguas las cinco dimensiones que constituyen la política energética de la UE, a saber: 1) la seguridad del suministro, 2) el mercado interior de la energía, 3) la eficiencia energética como contribución a la moderación de la demanda, 4) la descarbonificación del mix energético y 5) la promoción de la investigación y de la innovación. A raíz de este nuevo impulso integrador, las reticencias que han acompañado desde los inicios el desarrollo de la política energética europea ${ }^{99}$ deberían dejar paso a una Unión de la Energía tan prioritaria y esencial que la garantía del libre flujo de energía adquiriría el estatus de quinta libertad fundamental, sumándose a las libertades económicas de circulación de personas, mercancías, servicios y capitales ${ }^{100}$. Este propósito de las instituciones europeas es el salto cualitativo hacia la constitucionalización de la energía mediante su introducción en el núcleo de las libertades económicas que conforman la constitución material de la UE. Sin embargo, esto parece volver a proponer una constitución económica que intenta estabilizar la reproducción del sistema económico mediante su aseguramiento.

La nueva estrategia de la Unión de la Energía impugnará la potestad soberana estatal en materia de energía si se cumplen los propósitos de la Comisión de reformar los tratados para identificar las "regiones", en detrimento de los Estados, como motores de la nueva

\footnotetext{
${ }^{97} \operatorname{COM}(2015) 80$ final.
}

${ }^{98}$ Así lo califica con acierto TEFFER, P., "EU leaders want Energy Union: but what do they mean?", EUobserver, 20 marzo de 2015. Disponible en: https://euobserver.com/energy/128093

${ }^{99}$ Vid. HERRANZ SURRALLÉS, A., "La Unión Europea en la fragmentaria gobernanza global de la energía”, Barbé, E., La Unión Europea en las relaciones internacionales, Tecnos, 2014, p. 354.

${ }^{100}$ Ex multis, vid. PÉREZ DE LAS HERAS, B., El mercado interior europeo. Las libertades económicas comunitarias: mercancías, personas, servicios y capitales, Universidad de Deusto, 2004; GARRIDO MAYOL, V., GARCÍA COUSO, S. y ÁLVAREZ CONDE, E. (coords.), Comentarios a la Constitución Europea, vol. 2, Tirant lo Blanch, 2004; BARNARD, C., The Substantive Law of the EU: The Four Freedoms, OUP Oxford (4th edn), 2013. 
unión energética ${ }^{101}$. No parece que este marco favorezca la aparición de reglas y límites internos al sistema energético para hacer frente a los efectos externos de su funcionamiento; en otras palabras, la estrategia europea no conduciría a la "ecologización" del sistema energético inducida por presiones externas. Como hemos observado, el aumento de la demanda energética es una premisa para el funcionamiento del sistema económico y un corolario del sistema energético. Un cambio constitucional que integrara una perspectiva ecológica conllevaría modulaciones o produciría efectos en la demanda de energía en función de criterios de justicia. La justicia es, en efecto, la gran ausente en la comunicación sobre la Unión de la Energía ${ }^{102}$.

El plan político de las instituciones europeas y el marco normativo que tendrá que sustentarlo suscitan al menos tres preguntas para una futura agenda de investigación: 1) ¿cuál es el valor y la función de la Unión de la Energía?; 2) ¿la Unión de la Energía será solo una nueva dimensión reguladora del mercado interior de la UE o tendrá también una proyección exterior fundamental?; y 3 ) ¿la Unión de la Energía tendrá la capacidad de contribuir a la estabilización de la reproducción social en un futuro que no tiene antecedentes en términos ecológicos? Las respuestas a estas cuestiones permitirán desvelar la racionalidad del programa de integración, entender la estrategia y las relaciones de fuerza subyacentes del plan europeo para así valorar su consistencia en términos de eficacia, racionalidad, equidad y sostenibilidad. En otras palabras, las tres cuestiones planteadas permitirán analizar si se da un proceso de constitucionalización en los términos expuestos en el apartado anterior de este trabajo.

A la espera de ver cuáles serán los desarrollos institucionales y jurídicos, la estrategia apunta a una síntesis entre sistemas que generaría lo que Kjaer define como

\footnotetext{
${ }^{101}$ El vicepresidente de la Comisión para la Unión de la Energía, Maroš Šefčovič, declaró el pasado 1 de abril de 2015: "The era of the national energy mix is over. The era of the Energy Union has begun. And regions are the motor of that Union". Según los comentarios de VAN RENSSEN: "The idea is that regions will become the dominant decision-makers over energy policy in Europe in future. They will set regional renewables and energy efficiency targets, cooperate on infrastructure development, and most importantly, decide jointly on whether and how much energy is produced from coal, gas, oil, nuclear, biofuels, wind, solar etc.", VAN RENSSEN, S., "EU announces Treaty change to realise Energy Union", Energy Post, 1 de abril de 2015. Disponible en: http://www.energypost.eu/eu-announces-treaty-changerealise-energy-union/

${ }^{102}$ Con la salvedad de una referencia a la protección de los consumidores vulnerables y a la pobreza energética, cuestiones que los Estados deberían resolver mediante tarifas de solidaridad en el marco de un mercado cuyos precios no estén distorsionados por precios regulados.
} 
"functionally delineated economic constitution"103, que, sin embargo, no alcanzaría el nivel constitucional de estabilización que los sistemas del capitalismo avanzado requieren para su autolimitación a través de mecanismos reflexivos. La Unión de la Energía, en este momento, y utilizando un término de la estrategia de pensamiento de Foucault, es un "dispositivo" en el marco de la gubernamentalidad neoliberal, es decir, el conjunto de discursos, instituciones, estructuras, enunciados científicos, leyes y medidas administrativas, proposiciones morales y filosóficas, etc., el conjunto de prácticas discursivas y no discursivas que crea eventos y objetos y los hace gobernables. El dispositivo tiene naturaleza esencialmente estratégica y sirve para manipular relaciones de fuerza y dirigirlas, de acuerdo con una racionalidad, hacia una determinada dirección ${ }^{104}$. La estrategia de la Unión de la Energía se identifica con esta idea de dispositivo en la medida en que expresa una alteración de las relaciones de fuerza entre los Estados y la UE a favor de esta última sobre la base de una racionalidad que hemos identificado como propia del sistema económico. En esta estrategia se reclama el empoderamiento de los ciudadanos no por su condición de miembros de la sociedad política, sino como consumidores en el mercado. Esta construcción también descansa sobre la ideología neoliberal, tal como ha explicado Innerarity ${ }^{105}$. El constitucionalismo que surge con el derecho posmoderno puede actuar como contradispositivo, estableciendo límites, abriéndose a las diferentes racionalidades sociales y, en el ámbito que nos ocupa, fomentando la democracia y justicia energéticas, si se construye dentro del nuevo marco epistemológico del antropoceno.

\footnotetext{
${ }^{103}$ KJAER, P. F., "The Under-Complexity of Democracy", CALLIES, G.P., FISCHER-LESCANO, A., WIELSCH, D. y ZUMBANSEN, P., Soziologiche jurisprudenz festschrift für Gunther Teubner, De Gruyter-Verlang, 2009, p. 539.

${ }^{104}$ Vid. FOUCAULT, M., Dits et écrits, vol. III, pp. 299-300, citado y comentado por AGAMBEN, G., Che cos'è un dispositivo, I sassi, 2006.

105 “[...] neoliberal ideology has circulated a self- serving notion that is even harder to believe: that human beings are intelligent as consumers and stupid as voters. This thinking rests on Schumpeter's observation that the individual will is on firmer footing in the case of an individual's consumer decisions (given that the individual has direct experience of his choices), while the experience and will of the electorate are imprecise. Neoliberal economists have applied this principle of the wisdom of crowds to the marketplace, but the principle of the madness of the mob to politics. The conclusion that follows is disturbing: "If people are rational as consumers and irrational as voters, it is a good idea to rely more on markets and less on politics", vid. INNERARITY, D., "Shifting to intelligent societies", Higher Education in the World 5. Knowledge. Engagement and Higher Education: Contributing to Social Change, Palgrave Macmillan, 2013, pp. 8-9.
} 


\section{CONSIDERACIONES FINALES SOBRE ANTROPOCENO, GOBERNANZA, ENERGÍA Y CONSTITUCIONALISMO}

El cambio en las estructuras políticas y jurídicas que plantea la nueva estrategia de la Unión de la Energía ha ofrecido la oportunidad de reflexionar sobre cómo la cuestión energética penetra y se transforma en un momento esencial para el constitucionalismo en la época de la globalización. El discurso que se ha desarrollado ha tocado tanto cuestiones institucionales relacionadas con la soberanía, los Estados o el mercado como cuestiones teóricas sobre las normas jurídicas, la justicia, la ética o la cultura. Normalmente, estos temas son tratados asumiendo de forma implícita una perspectiva antropocéntrica. Sin embargo, a lo largo del presente trabajo se ha hecho hincapié en que la emersión de dinámicas de constitucionalización ${ }^{106}$ en el panorama fragmentado de los regímenes de gobernanza implica la capacidad del derecho de establecer "acoplamientos estructurales" con los sistemas sociales y el sistema ecológico. De esta manera, se observa que, más allá del espacio territorial de los Estados nacionales y de sus sistemas jurídicos, los procesos de constitucionalización (cognitivamente abiertos) permiten, en palabras de Teubner, poner límites a: “[...] las dinámicas incesantes, desconsideradas y destructivas de las racionalidades especializadas, no sólo de la economía capitalista, sino de cada sistema funcional, [que son] el fundamento de las catástrofes de la modernidad, de la enajenación de los individuos, de los devastadores conflictos sociales y del desastre ecológico". La aproximación holística a las diferentes aportaciones culturales debe necesariamente asumir también el cambio epistemológico derivado del antropoceno, la época geológica en la que se encuentra el planeta Tierra. En este sentido, las cuestiones constitucionales de la energía, del medio ambiente o de la economía, vistas a través de las lentes del antropoceno, brindan una nueva comprensión de las relaciones entra naturaleza y sociedad, y, por ende, un cambio hacia una nueva gubernamentalidad sobre la base de la "reinvention of the boundaries between nature and society and the political space for government intervention" ${ }^{\text {107 }}$. Al trascender las perspectivas institucionales y los ideales convencionales, la nueva época planetaria exige la reconsideración de la visión del espacio sociopolítico y jurídico mundial. Biermann y los demás científicos sociales involucrados en el Earth System

\footnotetext{
${ }^{106}$ Vid. KOTZÉ, L.J., “Arguing Global Environmental Constitutionalism”, Transnational Environmental Law, vol. 1, issue 1, 2012, pp. 199-233.

${ }^{107}$ LÖVBRAND, E., STRIPPLE, J. y WIMAN, B., "Earth System Governmentality Reflections on Science in the Anthropocene", Global Environmental Change, 19, 2009, pp. 7-13.
} 
Governance Project consideran que las evidencias científicas —según las cuales las actividades humanas están moviendo varios subsistemas de la Tierra fuera del rango de variabilidad típico de los últimos 500.000 años - apremian las sociedades a cambiar de rumbo y alejarse de umbrales críticos que, de ser traspasados, conllevarían cambios rápidos e irreversibles en el sistema Tierra. En palabras de Jaria, esta crisis ecológica impugna los fundamentos culturales de la modernidad y, por ende, los fundamentos de la organización del poder y de la creación del derecho ${ }^{108}$.

This requires fundamental reorientation and restructuring of national and international institutions toward more effective Earth system governance and planetary stewardship $[\ldots]$ We need [...] a "constitutional moment" in world politics and global governance ${ }^{109}$.

Desde la perspectiva de la "gobernanza del Sistema Tierra"110, procede repensar el término global más allá de la dimensión espacial, incluyendo sus vertientes temporales y causales; por lo tanto, según Kotzé, lo global hace referencia al "ecosistema político"111. Esta apertura hacia la politicización de las esferas sociales, alejándose de las teorías contractuales de la sociedad y de la presunta neutralidad de la técnica, estimula la constitucionalización y acentúa la necesidad de lograr una mayor democratización no solo a través de los procedimientos formalmente democráticos del sistema político, sino también mediante la democratización de los distintos ámbitos sociales $^{112}$. También del sistema energético, cuya relación con la democracia ha sido

\footnotetext{
${ }^{108}$ Para una teoría general de los fundamentos del derecho constitucional ambiental, vid. JARIA I MANZANO, La cuestión ambiental..., cit.

${ }^{109}$ BIERMANN, F. et ál., "Navigating the Anthropocene: Improving Earth System Governance", Science, vol. 335, 16 de marzo de 2012, pp. 1306-1307.

${ }^{110}$ Según Biermann et ál., se puede definir "Earth System Governance" como: “[...] the sum of the formal and informal rule systems and actor-networks at all levels of human society that are set up in order to influence the co-evolution of human and natural systems in a way that secures the sustainable development of human society [...]. Earth system governance covers more than problems of the 'global commons' [...] [it] [...] requires the integration of governance research at all levels. It must bridge scales from global to local", BIERMANN, F., "Earth System Governance as a Crosscutting Theme of Global Change Research", Global Environmental Change, 17, 2007, p. 329. Disponible en: http://www.glogov.org/images/doc/Biermann\%202007\%20GEC\%20Earth\%20System\%20Governance.p df. Vid. también BIERMANN, F. et ál., "Earth System Governance: A Research Framework", International Environmental Agreements: Politics, Law and Economics, 10, 4, 2010, p. 277-298. Disponible en: http://www.ivm.vu.n1/en/Images/Biermann\%20et\%20al\%20\%202010\%20INEA\%20ESG\%20Research\% 20Framework_tcm53-191335.pdf

${ }^{111}$ KOTZÉ, “Arguing Global...”, cit., p. 217.

112 TEUBNER, “Costituzionalismo societario...," cit., p. 65.
} 
por mucho tiempo infravalorada ${ }^{113}$. El éxito de esta tarea de constitucionalización y democratización depende del alejamiento del marco epistémico de la modernidad y de la instituciones del holoceno ${ }^{114}$. Estas últimas, según Dryzek, se han caracterizado por hacer caso omiso de los límites ecológicos y por el desacoplamiento entre sociedad y ambiente.

$[\ldots]$ the main political institution of the modern (late Holocene) era is the state. [...]As states took on more functions - notably for ensuring economic growth and providing social welfare - the non-human world was still ignored. [...] Many of the institutions that developed in the Holocene, such as sovereign states and capitalist markets, were complicit in generation of the unstable Earth system that now characterizes the Anthropocene. States have a priority for economic growth that subordinates ecological concerns, and a preoccupation with sovereignty that impedes global collective action. Capitalist markets for their part are equally addicted to material growth, and only recognize ecological constraints when forced to do so by non-market forces (such as government regulators) ${ }^{115}$.

El constitucionalismo que surge de los regímenes transnacionales, que en la teoría teubneriana se caracteriza por el acoplamiento estructural y la existencia de un vínculo entre los mecanismos reflexivos de tipo jurídico y los mecanismos reflexivos de otros sistemas, se verá reforzado por la reflexividad ecosistémica de la que habla Dryzek y que debería ser la primera virtud de las instituciones del antropoceno:

The ecosystemic dimension of reflexivity involves listening more effectively to an active Earth system, capacity to reconsider core values such as justice in this light, and ability to seek, receive and respond to early warnings about potential ecological state shifts ${ }^{116}$.

El discurso acerca de la constitucionalización de la energía se inscribe en esta nueva matriz del constitucionalismo ecosistémico global, que en palabras de Jaria constituye un sistema constitucional "que genera una nueva complejidad institucional que sustituye la exsclusividad del estado nacional como centro exclusivo de la imputación

\footnotetext{
${ }^{113}$ Sobre la relación entre la "maquinaria de la democracia" y el gobierno de la energía fósil, vid. MICHELL, T., "Carbon Democracy", Economy and Society, vol. 38, issue 3, 2009, p. 399-432, y del mismo autor, Carbon Democracy: Political Power in the Age of Oil, Verso, 2011.

${ }^{114}$ El holoceno es la época geológica de los últimos 10.000 años, que habría terminado hace aproximadamente unos 250 años, es decir, desde que hay evidencias del aumento de dióxido de carbono y metano en la atmósfera, dejando paso al antropoceno.

${ }^{115}$ DRYZEK, “Institutions...”, cit., pp. 2-3-5.

${ }^{116}$ Ibíd., p. 17.
} 
normativa" ${ }^{117}$. Esta matriz recupera la idea de los derechos constitucionales como límites al poder, esta vez no solo al poder público, sino también como límite al poder privado en el sistema del capitalismo avanzado, además de articular las garantías institucionales contra las amenazas de las matrices anónimas a los sistemas planetarios.

La estrategia europea de la Unión de la Energía no es un mero instrumento de regulación del mercado interior de la energía, sino una constitución económica material vinculada a una visión de gubernamentalidad neoliberal dotada de proyección geoestratégica, ya que, tal como se expresa en la Comunicación de la Comisión:

[...] la Unión Europea tiene que aumentar su capacidad para proyectar su poder en los mercados mundiales de la energía. Junto con sus principales socios, la Unión Europea se esforzará en conseguir un mejor sistema de gobernanza mundial de la energía, lo que dará lugar a unos mercados energéticos mundiales más competitivos y transparentes.

Contra ese dispositivo hegemónico, la justicia energética, como derivación de la justicia ambiental, debe constituirse como idea regulativa ${ }^{118}$ del metabolismo energético social y proyectar un nuevo haz de principios jurídicos constitucionales para la gobernanza sostenible en el antropoceno. Los estudiosos ya han comenzado a identificar estos principios $^{119}$ : responsabilidad y custodia, resiliencia y reflexividad, previsión y suficiencia, son algunos de ellos. Su análisis supera el alcance de este trabajo, pero plantea una de las cuestiones más complejas y fundamentales para un constitucionalismo llamado no solo a estabilizar los fenómenos sociales, sino también a influir en los parámetros de funcionamiento del sistema Tierra.

\footnotetext{
${ }^{117}$ JARIA I MANZANO, J., La identificació del dret aplicable en un context normatiu complex, ponencia presentada en el seminario "Diàlegs sobre la justícia i els jutges", organizado por el Centre d'Estudis Jurídics i Formació Especialitzada de la Generalitat de Catalunya, en Poblet, los días 11, 12 y 13 junio de 2014.

${ }^{118}$ Sobre la justicia ambiental como idea regulativa del funcionamiento interno del metabolismo social, vid. JARIA I MANZANO, "El constitucionalismo...", cit.

${ }^{119}$ ROBINSON, N.A., "Fundamental Principle of Law for the Anthropocene?", Environmental Policy and Law, vol. 44, issue 1-2, 2014, pp. 13-27.
} 


\section{BIBLIOGRAFÍA}

AGAMBEN, G., Che cos'è un dispositivo, Roma, I sassi, 2006.

BARNARD, C., The Substantive Law of the EU: The Four Freedoms, OUP Oxford (4th edn), 2013.

BECKER, C., Ethical Aspects of the Energy Issue: Toward a Sustainable Energy Future, 2011.

BIERMANN, F., "Earth System Governance as a Crosscutting Theme of Global Change Research", Global Environmental Change, 17, 2007.

BIERMANN, F. et ál., "Earth System Governance: A Research Framework", International Environmental Agreements: Politics, Law and Economics, 10, 4, 2010.

BIERMANN, F. et ál., "Navigating the Anthropocene: Improving Earth System Governance", Science, vol. 335, 16 de marzo de 2012.

BRESSER-PEREIRA, L.C., "The Global Financial Crisis and a New Capitalism?”, Levy Economics Institute Working Paper, núm. 592, 12 de mayo de 2010.

CABALlERO, R.J., FARHI, E. y GOURINCHAS, P.O., "Financial Crash, Commodity Prices, and Global Imbalances", Brookings Papers on Economic Activity, 2008.

COADY, D., PARRY, I., SEARS, L. y SHANG, B., "How Large Are Global Energy Subsidies?”, IMF Working Paper No. 15/105, 2015.

CRUTZEN, P. y STOERMER, E.F., “The Anthropocene”, IGBP Newsletter, 41, 2000.

DOUTHWAITE, R., "Degrowth and the supply of money in an energy-scarce world", Ecological Economics, vol. 84, 2012.

DRYZEK, J.S., "Institutions for the Anthropocene: Governance in a Changing Earth System", British Journal of Political Science, abril de 2015, pp. 2-3-5.

DUBASH, N.K. y FLORINI, A., "Introduction to the Special Issue: Governing Energy in a Fragmented World", Global Policy, vol. 2, special issue, septiembre de 2011.

- "Mapping Global Energy Governance", Global Policy, vol. 2, special issue, septiembre de 2011. 
DUCHENE, F., Jean Monnet: the first statesman of interdependence, New York, London, Norton, 1994.

EPSTEIN, G.A., Financialization and The World Economy, Edward Elgar Publishing, 2006.

FOUCAULT, M., Dits et écrits, vol. III, Paris, Gallimard, 1994.

FRATIANNI, M., Financial Crisis, Safety Net and Regulation, Invited lecture at the Annual meeting of the Società Italiana degli Economisti, Perugia, 24 de octubre de 2008.

GARRIDO MAYOL, V., GARCÍA COUSO, S. y ÁLVAREZ CONDE, E. (coords.), Comentarios a la Constitución Europea, vol. 2, Tirant lo Blanch, 2004.

GOLDTHAU, A. y SITTER, N., The Power of Paradigms: The EU and Global Energy Policy, 2012.

HEDBERG, A., "EU's quest for energy security. What role for Energy Union?", European Policy Centre, Policy Brief, 3 de marzo de 2015.

HELM, D., "The European framework for energy and climate policies", Energy Policy, 64, 2014.

- "The price of oil", Energy Future Network, Paper no. 6, 3 de diciembre de 2014.

HERRANZ SURRALLÉS, A., "La Unión Europea en la fragmentaria gobernanza global de la energía”, Barbé, E., La Unión Europea en las relaciones internacionales, Tecnos, 2014.

INNERARITY, D., "Shifting to intelligent societies", Higher Education in the World 5. Knowledge. Engagement and Higher Education: Contributing to Social Change, Palgrave Macmillan, 2013.

JARIA I MANZANO, J., "El constitucionalismo de la escasez (derechos, justicia y sostenibilidad)", Revista Aranzadi de Derecho Ambiental, núm. 30, 2015.

- Crisis ambiental y decisión política: la fragmentación de los espacios políticos, ponencia presentada en el XI Congreso AECPA, 2013.

- La cuestión ambiental y la transformación de lo público, Tirant lo Blanc, 2011. 
- La identificació del dret aplicable en un context normatiu complex, ponencia presentada en el seminario "Diàlegs sobre la justícia i els jutges", organizado por el Centre d'Estudis Jurídics i Formació Especialitzada de la Generalitat de Catalunya, en Poblet, los días 11,12 y 13 junio de 2014.

KALLIS, G., KERSCHNER, C. y MARTÍNEZ-ALIER, J., "The economics of degrowth", Ecological Economics, vol. 84, 2012.

KESSLER, O., "The same as it never was? Uncertainty and the changing contours of international law", Review of International Studies, 37, 2011.

KJAER, P.F., "Law nad Order whitinh and beyond National Configurations", Normative Orders Working Papers, 2/2010.

- "The Under-Complexity of Democracy", Callies, G.P., Fischer-Lescano, A., Wielsch, D. y Zumbansen, P., Soziologiche jurisprudenz festschrift für Gunther Teubner, De Gruyter-Verlang, 2009.

- “Three-dimensional Conflict of Laws in Europe", Zentrum für Europäische Rechspolitik ZERP-Diskussionpapier, 2, 2009.

KOTZÉ, L.J., “Arguing Global Environmental Constitutionalism”, Transnational Environmental Law, vol. 1, issue 1, 2012.

LEANDER, A. y VAN MUNSTER, R., "Private Security Contractors in the Debate about Darfur: Reflecting and Reinforcing Neo-Liberal Governmentality”, International Relations, vol. 21, núm. 2, junio de 2007.

LEGGET, G., The Energy of Nations. Risk blindness and the road to renaissance, New York, Routledge, 2014.

LÖVBRAND, E., STRIPPLE, J. y WIMAN, B., "Earth System Governmentality Reflections on Science in the Anthropocene", Global Environmental Change, 19, 2009.

MARISCAL BERASTEGUI, N., "Jean Monnet: Plan Schuman, federación y método", Cuadernos Europeos de Deusto, núm. 22, 2000.

MARKOWITZ, E.M. y SHARIFF, A.F., "Climate change and moral judgement", Nature Climate Change, vol. 2, abril de 2012.

MARTÍNEZ ARRANZ, A., "Carbon capture and storage: Frames and blind spots", Energy Policy, vol. 82, julio de 2015. 
MCGOWAN, F., "International Regimes for Energy: Finding the Right Level for Policy”, Scrase, I. y Mackerron, G. (eds.), Energy for the Future, Palgrave Macmillan, 2009.

MICHELL, T., "Carbon Democracy”, Economy and Society, vol. 38, issue 3, 2009.

- Carbon Democracy: Political Power in the Age of Oil, Verso, 2011.

MORATA, F. y SOLORIO SANDOVAL, I., "When 'green' is not always sustainable: the inconvenient truth of the EU energy policy", Carbon Management, 4, 5, 2013.

MORGAN, T., "Perfect Storm. Energy, Finance and the End of Growth", Strategy Insights, issue nine, Tullett Prebon, 2013.

PAKALKAITE, V., Competence Creep Through the Backdoor: EU Energy Regulation and Competition Policy, 5th Biennial ECPR Standing Group for Regulatory Governance Conference, Barcelona, 25-27 junio de 2014.

PARENTE, A., Principios de Derecho de la Energía, Pamplona, Aranzadi, 2010, p. 35 y ss.

PARENTEAU, P., "It's the Biosphere, Stupid", Burger, M. et ál., Rethinking Sustainability to Meet the Climate Change Challenge, en Environmental Law Reporter, vol. 43,2012

PÉREZ DE LAS HERAS, B., El mercado interior europeo. Las libertades económicas comunitarias: mercancías, personas, servicios y capitales, Universidad de Deusto, 2004.

- "Las políticas de seguridad energética en la Unión Europea y los Estados Unidos: desafíos globales y compromisos comunes en la transición hacia un modelo energético más sostenible”, Revista de Derecho Comunitario Europeo, núm. 47, 2014.

PRANDINI, R., “La costituzione del diritto nell'epoca della globalizzazione. Struttura del società-mondo e cultura del diritto nell'opera di Gunther Teubner", Teubner, G., La cultura del diritto nell'epoca della globalizzazione. L'emergere delle costituzioni civili, Armando Editore, 2005.

PROEDROU, F., "Rethinking energy security: An inter-paradigmatic debate", Policy Paper, Hellenic Foundation for European \& Foreign Policy, núm. 24, febrero de 2015. 
ROBINSON, N.A., "Fundamental Principle of Law for the Anthropocene?", Environmental Policy and Law, vol. 44, issue 1-2, 2014.

SHILLER, R., Las finanzas en una sociedad justa, Barcelona, Deusto, 2012.

SJÅFELL, B., "Sustainable Development, EU Law and Companies: The EU Law Framework for the Sustainable Companies Project", International and Comparative Corporate Law Journal, vol. 8, núm. 1, 2011.

SOVACOOL, B., "Exposing the Paradoxes of Climate Change and Energy Governance", International Studies Review, 16, 2014.

SOVACOOL, B. y DWORKIN, M., Global Energy Justice: Problems, Principles, and Practice, Cambridge, Cambridge University Press, 2014.

SOVACOOL, B. y FLORINI, A., "Examining the Complications of Global Energy Governance”, Journal of Energy \& Natural Resources Law, vol. 30, núm. 2, 2012.

SOVACOOL, B. y DWORKIN, M., "Energy Justice: Conceptual Insights and practical applications", Applied Energy, vol. 142, 2015.

STEVENSON, H., Institutionalizing Unsustainability: The Paradox of Global Climate Governance, Berkeley, University of California Press, 2013.

TALUS, K., EU Energy Law and Policy. A Critical Account, Oxford, Oxford University Press, 2013.

TEFFER, P., "EU leaders want Energy Union: but what do they mean?", EUobserver, 20 de marzo de 2015.

TEUBNER, G., "The Anonymous Matrix: Human Rights Violation By Private Transantional Actors", Modern Law Review, vol. 69, 2006, pp. 327-346.

- "A Constitutional Moment? The Logic of Hit the Bottom", Kjaer, P.F., Teubner, G. y Febbrajo, A. (eds.), The Financial Crisis in Constitutional Perspective. The Dark Side of Functional Differentiation, Hart Publishing, 2011.

- CCostituzionalismo societario e política del comune", Chignola, S., Il diritto del comune. Crisi della sovranità, proprietà e nuovi poteri costituenti, Ombre Corte, 2012.

- Constitutional Fragments. Societal Constitutionalism and Globalization, Oxford University Press, 2012. 
TUSK, D., "A united Europe can end Russia's energy stranglehold", Financial Times, 21 de abril de 2014.

VAN RENSSEN, S., "EU announces Treaty change to realise Energy Union”, Energy Post, 1 de abril de 2015. 